\title{
REMARKS ON THE LIFE AND RESEARCH OF ROLAND L. DOBRUSHIN ${ }^{1}$
}

\author{
R.A. MINLOS, E.A. PECHERSKY \\ The Dobrushin Mathematical Laboratory, Inst. for Problems of Information \\ Transmission, The Russian Academy of Sciences, 19 Bol'shoi Karetnyi Per. \\ GSP-4 Moscow 101447, Russia \\ YU. M. SUHOV \\ University of Cambridge, Statistical Laboratory, DPMMS \\ 16 Mill Lane, Cambridge CB2 1SB, UK \\ and \\ The Dobrushin Mathematical Laboratory, Institute for Problems of Information \\ Transmission, The Russian Academy of Sciences, 19 Bol'shoi Karetnyi Per. \\ GSP-4 Moscow 101447, Russia
}

(Received August, 1996; Revised October, 1996)

\begin{abstract}
The life and research work of Professor R.L. Dobrushin (1929-1995) had a profound influence on several areas of probability theory, information theory, and mathematical physics. The paper contains a biographical note, a review of Dobrushin's results, and a list of his publications.

Key words: Markov Chains, Central Limit Theorem, Shannon's Theorem, Gibbs' Random Field, Specification, Phase Transition, Hamilton's Equations, Hydrodynamical Limit, Queueing Network.
\end{abstract}

AMS (MOS) subject classifications: 01A, 34L, 60F, 60G, 60J, 60K, 82B, 94A, 94B, $94 \mathrm{C}$.

\section{A Foreword}

In the autumn of 1995, the mathematical community was shaken by the news from Russia that Roland Dobrushin had died of cancer on November 12 at the age of 66 . He was at the peak of his creative powers; a number of his papers were in print or preparation. It is impossible to know how many other works were in conception. We hope that his colleagues and pupils will be able to reconstruct at least some of his ideas. Dobrushin's energy during the last period of his life is also demonstrated by the extensive travel he undertook in 1995 when he was already seriously ill. Among other meetings, he attended the Conference in Mathematical Physics (Aragats, Armenia, May 1995); the 23rd Bernoulli Conference on Stochastic Processes and Their Applications (Singapore, June 1995); and the "Probability and Physics" Conference (Renkum, The Nether-

\footnotetext{
${ }^{1}$ This work has been supported in part by the Russian Foundation of Fundamental Research (Grant 01-96-00250), the EC Grant "Training Mobility and Research" under Project No. 16296 (Contracts CHRX-CT 93-0411 and ERBFMRX-CT 96-0075) and the INTAS Grant under Project "Mathematical Methods for Stochastic Discrete Event Systems" (INTAS 93-820).
} 
lands, August 1995). He was planning to spend the autumn of 1995 in the Schrödinger Institute in Vienna collaborating with several of his co-workers.

It is difficult to assess the scale of the loss to mathematics in general (and Russian mathematics in particular) occasioned by his death. Dobrushin's enormous contribution to modern mathematics is not confined to his publications. He was a man who generated a special mathematical aura. Everybody within his orbit who had the slightest talent for creating new mathematical results was quickly included in active and absorbing research. Such research was always deeply motivated (important for newcomers) and conducted to the highest standards. For many mathematicians the subject they began to work on with Dobrushin became their main topic of fruitful research for years, if not decades, to come. His ideas and views, like waves in water, percolated (and continue percolating) throughout the mathematical community, not always recognized as initiated by Dobrushin. Alas, the source of the waves is no longer with us.

A number of events dedicated to Dobrushin's memory have taken place or are planned, e.g., a session of the Moscow Mathematical Society, (April 1996), the conferences at the Schrödinger Institute (Vienna, September 16-20, 1996), and INRIA (Versaille-Rocquencourt, October 21-25, 1996). Obituaries and biographical articles $[3,9,35,62,70,98]$ have been published; a number of journals are to have special issues in his memory. The present paper is an attempt to describe some of his research contributions; we have tried to make the material accessible to a large probabilistic audience, maintaining at the same time the necessary level of mathematical rigor. We pay special attention to the origins of his main ideas and to a retrospective analysis of his methods. We believe that these are important issues that have perhaps not been discussed in detail in the literature so far. A brief biography is provided, where we focus on several aspects of his life. Dobrushin's personality had a huge impact on entire fields of research in Russia and abroad. We understand that our comments are inevitably one-sided and selective; it is impossible, within the limits of a single article, to analyze in depth his influence upon the modern state of research.

We also give a complete list of Dobrushin's published works. In the case of Dobrushin's papers originally published in Russian and officially translated to English, we refer to the year of Russian publication.

The references to the translated Russian papers by other authors are to their English translation. In general, while referring to the Russian papers, volumes, the names of the authors and the titles of the journals, periodicals and volumes are reproduced in the Russian transliteration, whereas the titles of the papers are given in the English translation. We apologize to the reader for possible divergency with other translated versions of the same Russian titles which may exist in the literature.

Commenting on the papers in which Dobrushin was a co-author, we give his name only (for which we apologize to his numerous co-workers). This is merely for the sake of unity of style. However, it should be noted that, at least in our experience, he was always the natural leader of a team, without being patronizing. His ideas almost always worked well, and his picture of the final result was astonishingly correct.

\section{A Biographical Note}

Dobrushin, who was of German, Jewish and Russian origin, was born on July 20, 1929, in Leningrad (now St. Petersburg). His parents died when he was a child and he was brought up by relatives in Moscow. His mathematical abilities were noted at school, but it is not known whether his school interests were confined to mathematics. However, it is a fact that he successfully took part in Mathematical Olympiads, a popular competition open to talented school 
children in which they had to solve specially selected and prepared questions (the term "olympiad problem" in Russian mathematical jargon describes a particular style of question at these competitions). An episode occurred when, in the course of solving an olympiad problem, Dobrushin had to use an axiom about a partition of a plane by a single line, which he was not aware of at the time. As a result, he wrote in his solution that, much to his embarrassment, he did not know what a straight line was, a remark noted by the examiners.

After finishing secondary school in 1947, Dobrushin applied for admission to the Department of Physics (Fiz-Fak) of Moscow State University (MSU). However, he failed to pass the entrance examination, although apparently not on the basis of his abilities or knowledge of the subjects. This was during the period of rising anti-Semitism in official propaganda. The Soviet authorities were particularly sensitive about admitting Jews to this department, where a large number of future nuclear scientists were trained.

Nevertheless, he was able to gain admission to the Department of Mechanics and Mathematics (Mekh-Mat) of MSU. From the beginning he took an active part in a student seminar series run by Dynkin. Here, he acquired a deep interest in probability theory and a particular probabilistic style of thinking which often distinguishes great scientists in the field. After graduating in 1952, Dobrushin was admitted as a research student, with Kolmogorov as supervisor. Once again, he had great difficulty in obtaining this studentship, for reasons unrelated to his research. It is well known that Kolmogorov had to use all his influence to have him admitted. Many excellent mathematicians who graduated from MSU at around the same time, did not get research studentships.

In 1955, Dobrushin completed and defended his Ph.D. thesis, "A Local Limit Theorem for Markov Chains." He was then given a position at the Probability Section of Mekh-Mat. In 1956, he was awarded the prize of the Moscow Mathematical Society for young mathematicians, a prestigious award (though modest in material terms) that marked many future celebrities of Soviet mathematics. Dobrushin's thesis improved a series of theorems of his predecessors, among whom one can mention Markov, Bernstein and Linnik.

The fifties were a time when information theory emerged and quickly progressed, following the works of Shannon. Dobrushin also became interested in this area. We can only guess what moved him in this direction. Such a decision might have been influenced by Kolmogorov who advised young mathematicians to work in the new fields of probability theorem. However, one could suppose that Dobrushin was attracted by a striking "critical point" phenomenon discovered by Shannon about the error probability in decoding a long message. Dobrushin studied general conditions under which such a phenomenon holds; as before, he found a concept that is essential for the validity of Shannon's theorems, the so-called information stability. Working on these problems, he spent a large part of his time on propagating the ideas and methods of information theory (he always took seriously the task of popularizing new ideas and was indefatigable in this capacity). He edited the information theory section of Soviet Mathematical Reviews and began running a seminar series in the recently created Institute for Problems of Information Transmission (IPIT) of the then USSR (now Russian) Academy of Sciences. He ran this seminar series until his final days and took his duties extremely seriously. In 1962, he prepared and defended his doctorate on his results from Shannon's theory. His doctorate was awarded at the Moscow Institute of Applied Mathematics of the AS, where the mathematical part of the Soviet space program was developed at that time.

In the early sixties, Dobrushin felt that the subject of information theory was beginning to be exhausted, although he continued, with some interruptions, publishing papers in the field until the late seventies. According to his colleagues and friends, he had similar feelings about many areas of "classical" probability theory. Conceding that the whole stream of works in classical directions, rich in results and traditions, served an important purpose in constructing a unified theory, 
he came to the conclusion that focusing on traditional approaches somehow slowed down the development of completely new fields. He gave much thought to this problem and voiced his dissatisfaction with the situation to his colleagues. His frequent conversations with one of the authors of this paper (R.A.M.) were directed towards fundamental questions of statistical mechanics, in particular, the problem of phase transitions. In general, his intention was to find common ground between physics and probability theory (recall his attempt to be admitted to Fiz-Fak of MSU).

The second half of the fifties and the beginning of the sixties saw the start of a political thaw which, however incomplete and contradictory, irreversibly changed people's outlooks and created a spirit of independence and in many cases, defiance of official doctrines. The future dissident movement was founded in this spirit of defiance, as well as a general nonconformist attitude widely popular among scientists, writers, painters, and other members of the intelligentsia, especially in Moscow and Leningrad. However, the regime was still a powerful structure and it had many supporters who, for one reason or another, were prepared to oppose changes and close their eyes to repressive measures against those critics of the system who dared to go too far. Dobrushin had a socially active mind and a very strong and independent personality. Together with his deep conviction that democratic principles should be introduced into Russian society, this inevitably put him on a collision course with officialdom and its supporters. The story of his confrontation with the huge repressive machine is worth a separate article; here we mention the facts only.

In the autumn of 1956, a group of Mekh-Mat students made public a few copies of a typewritten literary bulletin. An early example of samizdat, there were among its authors and distributors names that left their mark on the future development of Russian mathematics. From a contemporary viewpoint, the bulletin's contents were innocuous. They included a speech by a popular Soviet writer in which he criticized several of his colleagues hiding an obvious lack of talent behind the orthodoxy of "socialist realism", excerpts from John Reed's essay on Trotsky (who, until the Fall of 1991, was considered a political evil of Soviet history), and a number of verses by young poets denied publication in the tightly controlled official magazines. The authors of the bulletin were perhaps naive in thinking that the time of long-awaited freedom had arrived.

This made the Mekh-Mat authorities nervous. The Soviet Army had just invaded Hungary to crush reform, and there was a danger of confrontation in the Middle East over Nasser's nationalization of the Suez Canal. In this situation, it was decided that the bulletin should be treated as an "outside enemy's" activity. (It should be noted that some prominent Makh-Mat members were outraged by the bulletin, primarily not because they disagreed with its contents or with the fact of its distribution, but because they were afraid of official repressions against the department.)

The departmental authorities summoned a meeting of the staff and students with the declared objective of "condemning" and "punishing" the "moral mutineers", but in reality to demonstrate "unanimous approval" of the official line at this complicated period. Such meetings were part of a long tradition in Soviet political life, and their scenario was tested and improved countless times, although in post-Stalin times, enthusiasm for condemnation was not as great as it had been. Speakers, carefully selected by the organizers in advance, duly denounced, with various degrees of histrionic severity, the heretics, and some of the accused demonstrated various degrees of repentance. However, the planned course of the meeting was disrupted by Dobrushin's speech, in which he declared that the bulletin was a manifestation of the eternal principles of freedom, and the authorities could only benefit if everybody were free to speak their mind. The absurdity of the proceedings immediately became clear to all present, and the authorities, to their outrage, lost control of the meeting.

However, Dobrushin's words cost him (and not only him) dearly. The local Communist Party bosses insisted that he should be fired. This was opposed by Kolmogorov, but even his influence had limits. There was no way for Dobrushin to be promoted, and he was not permitted 
to travel abroad, except to some countries under Soviet domination. (The system invented a special term for such a category of people; very loosely it can be translated as "non-voyager", more precisely, "voyager to Socialist countries exclusively.") All this could not prevent him from continuing to defy the authorities. He was a co-signatory of almost all the protest letters that circulated in Moscow in the 1960s.

With time, the repressive side of the regime prevailed, and Dobrushin's position at MSU became precarious. At the beginning of 1967, he left Mekh-Mat and accepted an offer from the Institute for Problems of Information Transmission. He organized a laboratory at IPIT and worked as its head until his death. The main direction of research in his laboratory was initially information and coding theory. He later added the theory of complex stochastic systems which embraced his emerging interests in statistical mechanics and queueing network theory (see below). He also taught part-time at the Moscow Physical and Technical Institute (Fiz-Tekh) where he held a professorship from 1967 to 1992 . He took an active part in editing Problems of Information Transmission which under him, became a well-known and respected journal. It must be mentioned that the leadership of IPIT showed great courage in giving him such a prominent position, partly explained by the different atmosphere prevailing in many institutes of the Academy of Sciences (AS). There was traditionally a strong liberal spirit in these places, and numerous dissidents and refuseniks enjoyed the loyalty of colleagues and the administration. There was also a general inertia of the machinery of repression which made for greater freedom.

As the head of the laboratory, Dobrushin showed an extraordinary ability to recruit talented young mathematicians and direct their work in a wide variety of problems. The climate he created was extremely favorable for genuine intensive research and encouraged mutual sympathy and friendship between the staff. Despite its relatively small size (about ten people), the laboratory achieved prominence in several fields of mathematics. One member was awarded a Fields Medal, another received the prize of the European Union of Mathematicians, a third, the distinguished prize of the IEEE. In general, Dobrushin's presence always created an atmosphere of good spirits, a desire to learn and produce new work, and a readiness to help and share with others.

The period from the mid-sixties was the golden age of Dobrushin's research career. Without interrupting his work in information theory, in 1963, he along with Minlos, introduced a seminar series at MSU, with the general aim of bringing statistical mechanics into the context of probability theory. The following year, they are joined by Sinai and, for a short period, by Berezin and Schwartz, and later still, Malyshev. The seminar series on statistical physics ${ }^{2}$ became a forum for intensive discussion of various problems in the new field and quickly gained an international reputation. A number of essential probabilistic concepts and constructions were created here which describe the phenomena of statistical mechanics. Dobrushin's main achievements during 19651970 were the concepts of a specification and of a Gibbs' random field. He understood that one of the most important phenomena of interest in statistical physics, phase transition, is described as a non-uniqueness of a Gibbs' field with a given specification. He then gave a short and beautiful proof of the existence of phase transitions in the Ising model and its modifications in dimensions two and higher, and went further by investigating the structure of the set of pure phases in these models. His main results in this direction are published in [1965a, 1966a, 1968a-c, 1970a-b, 1972a-b, 1973a-b, 1974c]. In our view, these papers are important not only because they laid the foundation of the modern equilibrium statistical mechanics and solved a number of difficult problems, but also (and perhaps mainly) because they contain or lead to many open questions which we are sure will inspire future waves of research. It can be observed that many of his later works inevitably became technically much more involved and less accessible for a wide audience.

Dobrushin's results of 1968-1975 became instantly famous and attracted crowds of new researchers from across the world. There were countless conferences and symposia and mutual

\footnotetext{
${ }^{2}$ In this context, the terms statistical physics and statistical mechanics are interchangeable.
} 
visits where his theory was discussed at length and in detail. However, the author himself was not able to put his foot beyond the Iron Curtain, although he was inundated with invitations. Instead, the stream of scientists from the Benelux countries, France, Germany, Italy, Japan, the Scandinavian countries, Switzerland, the United Kingdom, and the United States came to see Dobrushin in Russia or Soviet block states. Dobrushin's case became a headache for Soviet officials, but the system remained adamant. A curious episode occurred when the papers about a planned visit of an American colleague to IPIT arrived in the Institute during a very busy time. The visitor's name was misspelled in the course of translation to Russian, and Dobrushin in a hurry did not recognize him. Consequently, the Institute did not give its approval and the visit was canceled. In traditions of Cold War, the real cause of canceling the visit was not made known to the American colleague, who suspected that the Soviet repressive institutions prevented Dobrushin not only from going abroad, but also from meeting foreigners in his own country (such measures were sometimes applied to people who fell in disgrace under the Soviet regime.) The colleague gave an interview to Voice of America which was subsequently broadcast to the Soviet Union. (In these days, the broadcasts from the West were regularly listened to by many in the USSR. It was an alternative source of news to the official Soviet media.) Learning about the broadcast, Dobrushin remembered the case. It took another year to make the visit possible, but in the end, everyone was happy.

Working in an institute which specialized in the study of various aspects of information transmission. Dobrushin naturally continued his own interest in these areas. By the mid-seventies, his attention was mainly focused on problems in queueing network theory. Here, the object of study is a collection of servers that process a "flow" of tasks (which depending on the context may be messages, calls, programs, etc.) according to certain rules. The problems lie in assessing delays in processing the tasks, loss probabilities, non-overload conditions, etc. Dobrushin approached these problems by using analogies with objects from statistical physics. His influence in this field went far beyond his published works and may be traced in numerous papers by his followers.

From 1988 when the Soviet Union entered the final phase of perestroika, Dobrushin was allowed to travel without restrictions. With the change of political regime, he was also accepted back at Moscow State University, where from 1991 to his death, he held a part-time professorial position at the Probability Section of Mekh-Mat. In general, the character of research in the USSR in many fields of mathematics and theoretical physics changed dramatically at that time. The number of visiting scientists from the West went down whereas the opposite stream of visitors from the Soviet Union became much more intense. The number of trips abroad and their duration were considered by many as a sign of reputation and became a matter of competition. The worsening economic and social situation forced the emigration, temporary or permanent, of leading and prominent specialists in practically all fields. The famous Moscow seminar series went through hard times, and many of them ceased altogether. This was the case with the seminar series in statistical physics. It continued with interruptions until 1994 and was then terminated.

In this situation, Dobrushin was one of the few whose enthusiasm remained constant. He was a profound optimist by nature. Despite numerous offers, he never sought a permanent position in the West although in 1994, he accepted an invitation to spend up to six months a year at the Schrödinger Institute in Vienna. He traveled widely, but was always glad to return to Moscow. He loved the city and the country, whatever it was called and whichever political force was in power. After a period of protest in the sixties, he was not directly involved in any political activity, but continued to be deeply interested in politics, both inside Russia and abroad. He was a great reader of journals and general and political magazines (e.g., during the Soviet era, he regularly read Marxists magazines printed by leftist parties and groups in the West which he, at some risk, managed to obtain from foreign friends and colleagues and kept in his flat). He evidently had a very good understanding of the disposition of political forces. His predictions of political events were always amazingly accurate. 
Dobrushin's academic career at home developed in line with his status as a "non-voyager." The Mathematics Section of the USSR and then Russian AS did not elect him either a full member or a member-correspondent; his candidacy was not even seriously discussed during election campaigns. (To be precise, he never sought the election.) Despite his fame and reputation, he was treated as an outsider by the Soviet mathematical officialdom, as were many other outstanding mathematicians of his time. Partly this was due to anti-Semitism, partly to the servility towards the Soviet system of some Academy members, and partly to the internal rivalry between different groups of academicians. Dobrushin's own anti-establishment attitude did little to help him to become more popular with the Soviet academic elite. His staunch reformist convictions were once more demonstrated in this address to the General Assembly of the USSR AS in March 1990, at the high time of political debates on the future of the Soviet system in general and the particular role of the Academy. This speech was enthusiastically greeted by the large part of the audience which included young researchers but was met with skepticism by the conservative part of academicians.

In 1982, Dobrushin was elected an Honorary Member of the American Academy of Fine Arts and Sciences in Boston. High officials of the Soviet Academy of Sciences urged him to decline the honor (it was the peak of the last period of confrontation of superpowers), but Dobrushin refused to follow their "advice." In 1993, he was elected an Associated Foreign Member of the USA National Academy and in 1995, a Member of the European Academy.

Dobrushin served as a member of the editorial or advisory boards of Communications in Mathematical Physics, Journal of Statistical Physics, Theory of Probability and Its Applications and Selecta Mathematica Sovietica. He also edited a number of volumes of research papers of Russian authors, both in Russian and English.

From 1991, Dobrushin increased the number of staff in his laboratory at IPIT, and greatly extended its research. It is now called the Dobrushin Mathematical Laboratory and carries out research in a number of directions into information and coding theory, queueing network theorem, mathematical physics and representation theory.

\section{A Survey of Dobrushin's Research History}

\subsection{Markov processes}

In his first series of published papers, Dobrushin studied non-homogeneous Markov chains. The main problem he was interested in is the central limit theorem (CLT) for this class of processes. As mentioned above, he invented a specific parameter known as the ergodicity coefficient which describes a degree of "homogeneity" of a general Markov chain. Consider an array of random variables

$$
\begin{gathered}
X_{1}^{(1)}, X_{2}^{(1)}, X_{3}^{(1)}, \ldots \\
X_{1}^{(2)}, X_{2}^{(2)}, X_{3}^{(2)}, \ldots, \\
\ldots \\
X_{1}^{(n)}, X_{2}^{(n)}, X_{3}^{(n)}, \ldots \\
\ldots
\end{gathered}
$$

Each row $X_{1}^{(n)}, X_{2}^{(n)}, X_{3}^{(n)}, \ldots$ forms a Markov chain with the transition probabilities $P_{t}^{(n)}(a, A)=\mathbb{P}\left(X_{t+1}^{(n)} \in A \mid X_{t}^{(n)}=a\right)$. The ergodicity coefficient $\alpha_{n}$ of the $n$th row is defined by

$$
\alpha_{n}=\min _{1 \leq t \leq n}\left[1-\sup _{a, b, A}\left|P_{t}^{(n)}(a, A)-P_{t}^{(n)}(b, A)\right|\right] .
$$


Dobrushin showed that

If the random variables in (1) are uniformly bounded and

$$
\alpha_{n} n^{1 / 3} \rightarrow \infty
$$

then for array (1) the CLT holds true.

This result concluded a series of papers opened by a 1910 paper by Markov [63] who proved the CLT for two-state chains under the condition

$$
\alpha_{n} \geq \alpha>0
$$

The next step was made by Bernstein [12-14] who subsequently, in 1922, 1926 and 1928, obtained the CLT, still in the case of two states, when

and

$$
\begin{aligned}
& \alpha_{n} n^{1 / 7-\epsilon} \rightarrow \infty, \\
& \alpha_{n} n^{1 / 5-\epsilon} \rightarrow \infty,
\end{aligned}
$$

$$
\alpha_{n} n^{1 / 3-\epsilon} \rightarrow \infty
$$

for any $\epsilon>0$. In 1947, Sapogov [88] obtained the result where the chain again has two states and obeys (3). For a general state-space, the CLT was proved under condition (5a) in [15] and (5b) in [88]. For an arbitrary finite state-space it was proved under (6) by Linnik [60] in 1948. Dobrushin [1956a-c] proved the CLT under condition (3) for a general "bounded" state-space (e.g., an interval of a real line).

Dobrushin's result may be treated as final (or close to final) because Bernstein [15] gave an example in which $\alpha_{n} n^{1 / 3} \rightarrow 0$ where the CLT does not hold (Dobrushin himself constructed an example where the state-space of the Markov chain is the real line and the limiting law is not Gaussian, but stable). It is worth noting that the previous statements were less elegant since they did not use directly the ergodicity coefficient. As may be seen from the definition, $\alpha_{n}$ assesses how different the probabilities of transition are, from different states into the same set; the CLT holds when such a difference is not too large. In the proof of the CLT, Dobrushin uses martingales; at that time such an approach was yet not widely popular. The idea used his papers may be described by the word "contractivity." In one form or another it was successfully used many times in his subsequent papers.

Apart from the CLT, Dobrushin studied other problems from Markov chain theory. He obtained necessary and sufficient conditions for the number of jumps of a non-homogeneous Markov process to be finite [1954a]. He constructed an example of a Markov process with countably more states each of which is instantaneous [1956d]. In a short note [1955b] he proved asymptotical normality of the time that a symmetric random walk spends in a subset of $\mathbb{Z}$. Paper [1956e] was a harbinger of his future interests. Let $\Psi_{0}$ be a translation-invariant random point field in $\mathbb{R}^{d}$ with a finite rate $\rho$. Assume that during the unit time each particle performs a random jump, and the displacements of different particles are i.i.d., with probability density $p(x), x \in \mathbb{R}^{d}$. Then the random field $\Psi_{n}$ generated at time $n$ converges "weakly" to a homogeneous Poisson point field $\Phi$ with rate $\rho$.

At that time paper [1956e] seemed rather atypical and did not give rise to systematic work in this direction. However, the concept of an infinite particle system that emerged from this paper had serious impact on his later research. From the end of the fifties onwards Dobrushin focused on problems of information theory.

\subsection{Information theory}

One of the main problems of coding theory is: Given a "noisy" channel of information trans- 
mission, encode a text at the input port and decode it at the output port so that the error probability becomes negligible (or at least minimal). More precisely, suppose that a total of $M$ different "messages" is given, which are to be transmitted through a channel. To each message one assigns a distinct codeword that is a sequence of $n$ binary digits, $n \geq \log _{2} M$. The collection $\mathcal{H}\left(=\mathcal{M}_{n}\right)$ of codewords is called a code (of length $n$ ); it is a subset of the set $\{0,1\}^{n}$ of $2^{n}$ possible binary $n$-words.

In the course of transmission random errors occur; to start with, we assume that the statistics of the channel is known, i.e., we know (for any $n$ and any $a \in \mathcal{M}$ and $b \in\{0,1\}^{n}$ ) the probability $P(a \mid b)\left(=P^{(n)}(a \mid b)\right)$ to receive a word $a$ given that a codeword $b$ has been sent. A popular example is a "memoryless" channel, where $P(a \mid b)=\prod_{i=1}^{n} w\left(a_{i} \mid b_{i}\right), a=\left(a_{1}, \ldots, a_{n}\right), b=$ $\left(b_{1}, \ldots, b_{n}\right), a_{i}=0$ or 1 ; here, $w\left(a_{i} \mid b_{i}\right)$ is the probability of receiving binary symbol $a_{i}$ when $b_{i}$ has been sent.

Suppose we use the maximum likelihood principle for decoding, according to which the received word $a$ is decoded by the codword $b^{*}$ that maximizes $P(a \mid b)$ in $b \in \mathcal{M}$. (There exist both rigorous and informal arguments in favor of such a decoder.) Call a number $R \in[0,1)$ a reliable transmission rate (TR) if it is possible to vary $\mathcal{H}$ and $n$ so that $(i) M, n \rightarrow \infty,(i i)$ the $\operatorname{ratio} \frac{\log M}{n}$ (called the transmission rate of code $\mathcal{M}$ ) remains constant and equals $R$ and (iii) the error-proba bility $\max _{b \in \mathcal{N}}\left[\sum_{a: a \neq b} P(a \mid b)\right]$ vanishes in the limit. Call the supremum $C=\sup [R: R$ a re liable TR] the channel capacity. Then, by definition, the error-negligible transmission is possible for $R<C$ and not for $R>C$. How may one assess $C$ or at least check that $C>0$ ? The answer was given by Shannon in 1948; we state it informally, following the original paper [89].

There exist a lower bound for $C$ (direct Shannon's theorem) and an upper bound (converse Shannon's theorem), in terms of an asymptotical behavior of $P(\cdot \mid \cdot)$ as $n \rightarrow \infty$. Under "natural" assumptions on $P(\cdot \mid \cdot)$ (e.g., for a memoryless channel) the lower and upper bounds coincide (and may be calculated in terms of $w(\cdot \mid \cdot)$ ); moreover, for $R<C$ the error-probability decays exponentially.

As noted, Shannon result produced a strong impression at the time, in particular the existence (and a deep "physical" significance) of a "critical" value $C$. In his initial papers in information theory, Dobrushin studied the possibility of extending Shannon's theorems to a more general set-up where the coding alphabet is arbitrary. He invented the above condition of information stability which turns out to be sufficient for Shannon's theorems to hold. He later extended the theory to the case where the channel statistic is not known. Here is the result of [1975b].

Assume that the channel used for transmission is memoryless, but the symbol-to-symbol transition probability $w(\cdot \mid \cdot)$ is not given (e.g., may vary from time to time); one knows only that it belongs to a certain class $W$. The input symbols $b_{i}$ are from an "alphabet" $X$ and the output ones from $Y$; both $X$ and $Y$ are supposed to be finite (in the above set-up, $X=Y=\{0,1\}$ ). Given a probability distribution $p\left(=p_{X}\right)$ on $X$ and $w \in \mathcal{W}$, set

$$
\begin{gathered}
I(p, w)=\sum_{y \in Y} \frac{\sum_{x \in X} p(x) w(x, y) \log w(x, y)}{\sum_{z \in X} p(z) w(z, y)}, \\
C_{p}\left(=C_{p}(W)\right)=\min _{w \in \bar{W}} I(p, w),
\end{gathered}
$$

where $\bar{W}$ is the closed convex hull of $\mathcal{W}$, and

$$
C(=C(\mathscr{W}))=\max _{p} C_{p}
$$

Suppose that at the input of the channel one has words $a=\left(a_{1}, \ldots, a_{n}\right)$ from $X^{n}$; the total number of the input messages, as before, equals $M$. A code (more precisely, a coder-decoder pair) is 
defined as a set

$$
\mathcal{A}=\left\{\left(a^{(i)}, B_{i}\right): i=1, \ldots, M\right\}
$$

where $a^{(i)} \in X^{n}, B_{i} \subset Y^{n}$ and $a^{(i)} \neq a^{(j)}, B_{i} \cap B_{j}=\emptyset, 1 \leq i<j \leq M$. If one receives a word $b \in B_{i}$, it is decoded as $a^{(i)}$. The average error probability, given that the channel statistic, while transmitting the $s$ th digit, is determined by $w_{s} \in \mathcal{W}, s=1, \ldots, n$, equals

$$
e(\mathcal{A}, \mathbf{w})=\frac{1}{M} \sum_{i=1}^{M} \sum_{\left(y_{1}, \ldots, y_{n}\right) \in B_{i}} \prod_{s=1}^{n} w_{s}\left(a_{s}^{(i)}, y_{s}\right), \mathbf{w}=\left(w_{1}, \ldots, w_{n}\right) .
$$

The average error-probability of code $\mathcal{A}$ for a given class $\mathscr{W}$ is

The $[1975 \mathrm{~b}]$ theorem states:

$$
\mathbf{e}(A)=\sup _{\mathbf{w} \in \mathscr{W}^{n}} e(\mathcal{A}, \mathbf{w})
$$

For any probability distribution $p$ on $X$ satisfying some additional condition, for all $R<$ $C_{p}(\mathcal{W})$, there exists $\bar{\lambda}$ such that, for $0<\lambda \leq \bar{\lambda}$ and all $n$ large enough, there exists a code $\mathcal{A}$ of length $n$ and size $M=\left[2^{n R}\right]$ and with $\mathbf{e}(\mathcal{A}) \leq \lambda$. Conversely, for any $\gamma>0$, any $\lambda$ between 0 and 1 and all $n$ large enough there is no code of length $n$ and size $\left[2^{n(C(\mathscr{W})+\gamma)}\right]$ with $\mathbf{e}(\mathcal{A})<\lambda$.

We do not go into detail of the additional condition on $p$. This condition is not just technical. [1975b] gives examples where both the condition and the assertion of the theorem fail. However, this condition apparently excludes only some "degenerate" variety of cases.

The above results are only a part of Dobrushin's activity in information theory. We briefly mention some others:

(a) Dobrushin discovered (and used) an elegant formula for mutual entropy [1959b]:

$$
I((\xi, \eta), \zeta)+I(\xi, \eta)=I(\eta ;(\xi, \zeta))+I(\xi, \zeta)
$$

(b) He studied the capacity and $\epsilon$-entropy for some classes of channels and sources [1958c], [1963d].

(c) He established the dependence of the logarithm of the optimal error-probability as a function of code length [1960c], [1962b].

(d) He studied sequential decoding [1964a].

Dobrushin's last work [1987c] which was strong connections with information theory was devoted to the $\epsilon$-entropy of Gibbs' random fields for high temperatures.

\subsection{Equilibrium statistical mechanics and the theory of random fields}

The problem Dobrushin focused on at the beginning of the sixties was the construction of probabilistic models of matter (gas or solid) exhibit the phenomenon of phase transitions. An important role in his studies was played by [75] where a "physical" argument is developed showing that the Ising model of a lattice ferromagnet should exhibit a phase transition when the dimension of the lattice is two or higher. Notable publications were [81-83], developing useful tools for describing a state of an infinite physical system. Dobrushin was of course, aware of the famous works [74] and [44] (and a number of later papers, e.g., [58]) giving the exact solution of the twodimensional Ising and related models. However, he deliberately (and somewhat demonstratively) avoided the route of exact solutions, believing a qualitative theory to be the true path towards understanding complicated phenomena. This attitude was typical of him in other fields of research.

In [1965a, b] and [1966a] Dobrushin produced the first (and rather complicated) version of the proof of the existence of phase transition in the Ising model dimension two and higher. Alternative or similar arguments and proofs were given in $[10,24,30]$ and [67]. In [1968a] 
Dobrushin developed a general approach to the concept of phase transition as the non-uniqueness of a random field with a given system of conditional probabilities (or a given specification, as it was later called). In [1968b, c] and [1969a] he put this problem in the context of various models in statistical mechanics and in particular gave a new proof, short and beautiful, of the existence of phase transitions in Ising-type models. It is this proof, refining the original Peierls argument, which is now presented in most textbooks and reviews to demonstrate the phenomenon of phase transitions. Additional chapters in $[1970 \mathrm{a}, \mathrm{b}]$ extend this theory. Together with papers [20, 56, $57,82,83]$ and [86] (see also [64-66]), these papers formed the foundation for further rapid development of the probabilistic approach to the equilibrium statistical mechanics (and later on, Euclidean quantum field theory). See [22, 25, 61, 85, 91, 94].

The main tool to describe a statistical mechanics model is the above-mentioned concept of specification. Fix a finite set $X$ and consider the $X$-valued functions $\sigma: \mathbb{Z}^{d} \rightarrow X$ on a $d$-dimensional cubic lattice $\mathbb{Z}^{d}$. Any such function is treated as a configuration of "spins" on $\mathbb{Z}^{d}$; the value of $\sigma(t)$ gives a state of the spin assigned to the lattice site $t \in \mathbb{Z}^{d}$. Similar definitions hold when $\mathbb{Z}^{d}$ is replaced by its subset. In the Ising model $X=\{-1,+1\}$; the value $\sigma(t)=+1$ corresponds to the + and $\sigma(t)=-1$ to the - direction of a spin at $t \in \mathbb{Z}^{d}$. The space of the configurations on $\mathbb{Z}^{d}$ is the Cartesian product $X \mathbb{Z}^{d}$. It is endowed with a natural metric, and one considers the probability measures on the corresponding Borel $\sigma$-algebra $\mathfrak{B}$. Any such probability measure $\mathbb{P}$ is a random field $(\mathrm{RF})$ on $\mathbb{Z}^{d}$. According to Kolmogorov's theorem, it is given by a consistent family of "finite-dimensional" probability distributions $P^{\Lambda}$ on $X^{\Lambda}$, where $\Lambda \subset \mathbb{Z}^{d}$ is an arbitrary finite set of lattice sites (for many purposes it is enough to consider lattice "cubes" $\{-N,-N+1, \ldots$, $\left.N-1, N\}^{d}\right)$.

A specification is defined as a consistent family of conditional probability distributions $P^{\Lambda}\left(\cdot \mid \bar{\sigma}_{\Lambda^{c}}\right)$ on $X^{\Lambda}$, given a configuration $\bar{\sigma}_{\Lambda^{c}}$ on the complement $\Lambda^{c}=\mathbb{Z}^{d} \backslash \Lambda$ (called a boundary condition for $\Lambda$ ). A natural "physical" way to define such a family is to $(a)$ fix a collection of functions

$$
\Phi_{\Lambda}: X^{\Lambda} \rightarrow \mathbb{R}^{1}
$$

(b) define functions of two variables $\sigma_{\Lambda} \in X^{\Lambda}$ and $\bar{\sigma}_{\Lambda^{c}} \in X^{\Lambda^{c}}$ by

and $(c)$ set

$$
H_{\Lambda}\left(\sigma_{\Lambda} \mid \bar{\sigma}_{\Lambda^{c}}\right)=\sum_{\tilde{\Lambda}: \tilde{\Lambda} \cap \Lambda \neq \emptyset} \Phi_{\tilde{\Lambda}}\left(\sigma_{\Lambda \cap \tilde{\Lambda}} \vee \bar{\sigma}_{\tilde{\Lambda} \backslash \Lambda}\right)
$$

$$
P^{\Lambda}\left(\sigma_{\Lambda} \mid \bar{\sigma}_{\Lambda^{c}}\right)=\frac{1}{Z_{\Lambda}\left(\bar{\sigma}_{\Lambda}{ }^{c}\right)} \exp \left[-H_{\Lambda}\left(\sigma_{\Lambda} \mid \bar{\sigma}_{\Lambda^{c}}\right)\right]
$$

As before, $\Lambda$ (and $\tilde{\Lambda}$ ) runs here over finite subsets of $\mathbb{Z}^{d} ; \sigma \sigma_{\Lambda \cap \tilde{\Lambda}} \vee \bar{\sigma}_{\tilde{\Lambda} \backslash \Lambda}$ in (15) is a configuration on $\tilde{\Lambda}$ formed by "joining" two configurations, $\sigma_{\Lambda \cap} \cap$ which is the restriction of $\sigma_{\Lambda}$ to $\tilde{\Lambda} \cap \Lambda$ and $\bar{\sigma}_{\tilde{\Lambda} \backslash \Lambda}$ which in turn is the restriction of $\bar{\sigma}_{\Lambda^{c}}$ to $\widetilde{\Lambda} \backslash \Lambda$ (the latter is empty when $\tilde{\Lambda} \subseteq \Lambda$, in which case $\sigma_{\Lambda \cap \tilde{\Lambda}} \vee \bar{\sigma}_{\tilde{\Lambda} \backslash \Lambda}$ is simply $\sigma_{\tilde{\Lambda}}$, the restriction of $\sigma_{\Lambda}$ to $\left.\tilde{\Lambda}\right)$. The quantity $Z_{\Lambda}\left(\bar{\sigma}_{\Lambda^{c}}\right)$ in (16) is a normalizing constant called the partition function with boundary condition $\bar{\sigma}_{\Lambda^{c}}$ :

$$
Z_{\Lambda}\left(\bar{\sigma}_{\Lambda^{c}}\right)=\sum_{\tilde{\sigma}_{\Lambda} \in X^{\Lambda}} \exp \left[-H_{\Lambda}\left(\tilde{\sigma}_{\Lambda} \mid \bar{\sigma}_{\Lambda^{c}}\right)\right] .
$$

All series in (15) are supposed to converge absolutely.

Pictorially, $\left\{\Phi_{\Lambda}\left(\sigma_{L}\right)\right\}$ is a family of "multi-body" interaction potentials contributing to a "conditional" energy $H_{\Lambda}\left(\cdot \mid \bar{\sigma}_{\Lambda^{c}}\right)$ in $\Lambda$ given boundary condition $\bar{\sigma}_{\Lambda^{c}}$; the sum in (15) describes the "energy" of interaction of spins $\sigma(t), t \in \Lambda$, constituting $\sigma_{\Lambda}$ plus the energy of their interaction with spins $\bar{\sigma}(\bar{t}), \bar{t} \in \Lambda^{c}$, constituting $\bar{\sigma}_{\Lambda^{c}}$. An important observation is that the distribution $P^{\Lambda}\left(\cdot \mid \bar{\sigma}_{\Lambda^{c}}\right)$ favors the configurations $\sigma_{\Lambda}$ with a minimal energy $H\left(\sigma_{\Lambda} \mid \bar{\sigma}_{\Lambda^{c}}\right)$ (in 
physical terminology they are called ground states). In the Ising model, $\Phi_{\Lambda}$ is non-zero only when $\Lambda$ is a one- or a nearest-neighbor two-point subset of $\mathbb{Z}^{d}$ :

$$
\Phi_{\Lambda}\left(\sigma_{\Lambda}\right)=\left\{\begin{array}{c}
-\mu(t) \beta \sigma(t) \text { if }|\Lambda|=1 \text { and } \Lambda=\{t\} \\
\beta J \sigma(t) \sigma\left(t^{\prime}\right) \text { if }|\Lambda|=2, \Lambda=\left\{t, t^{\prime}\right\}, \text { and }\left\|t-t^{\prime}\right\|-1 \\
0 \text { otherwise. }
\end{array}\right.
$$

The constant $\beta \geq 0$ is the inverse temperature of the system; the abundance of constants is used to analyze in detail the behavior of the model in various physical regions. The first line in (18) describes a one-body (or self-) interaction; physically, it corresponds to an external magnetic field, and the value $\mu(t) \in \mathbb{R}^{1}$ measures the strength of this field at site $t \in \mathbb{Z}^{d}$ (sometimes called the chemical potential at site $t$ ). When $\mu(t)>0$, the field favors the + and when $\mu(t)<0$, the - spin at site $t$. The second line describes a two-body or binary interaction (or coupling) of the pairs of nearest-neighbor spins ( \|\| stands for the Euclidean norm), and $J \in \mathbb{R}^{1}$ is called the coupling constant. When $J<0$, the interaction favors the pairs of neighbor spins to be of the same sign (in which case, the model is called ferromagnetic or attractive) whereas $J>0$ favors them to be of the opposite signs (in which case the model is called antiferromagnetic or repulsive).

Continuing further with ground states (GS), one can speculate that in the ferromagnetic case with a space-homogeneous magnetic field (i.e., $\mu(t) \equiv \mu$ ), the GS on the whole $\mathbb{Z}^{d}$ is $\sigma \equiv-1$ when $\mu \leq 0$ and $\sigma \equiv+1$ when $\mu \geq 0$; thus, in the absence of the magnetic fields when $\mu=0$, one has two "competing" GSs. See Figure 1.
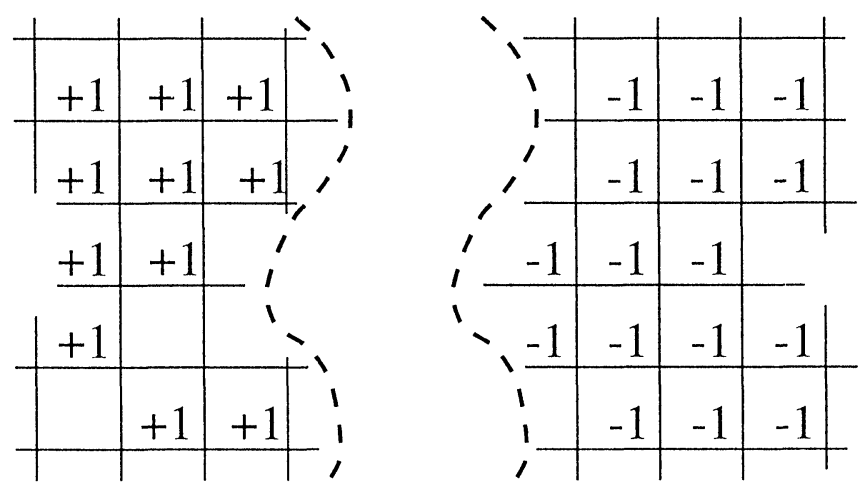

Figure 1

On the other hand, in the antiferromagnetic case with an alternating field $\left(\mu(t)=-\mu\left(t^{\prime}\right)\right.$ if $t$, $t^{\prime} \in \mathbb{Z}^{d}$ are nearest-neighbor) the GS is a "chessboard" configuration where $\sigma(t)$ has the same sign as $\mu(t)$. Again, the case $\mu(t) \equiv 0$ leads to two GSs that are distinguished by $\sigma(O)$, the value assigned to the origin $O$. (See Figure 2.) Furthermore, in both cases, in the absence of the magnetic field, the competing GSs exhibit a symmetry. They are transformed into each other by "flipping" the values of the spin (i.e., changing them to their opposites) at each lattice site. Finally, in the ferromagnetic model, the GSs are translation-invariant, whereas in the antiferromagnetic one translation-periodic and transformed into each other by a unit space shift. (In fact, the above configurations do not exhaust the set of (suitably defined) GSs, but they suffice for our immediate purposes.) 

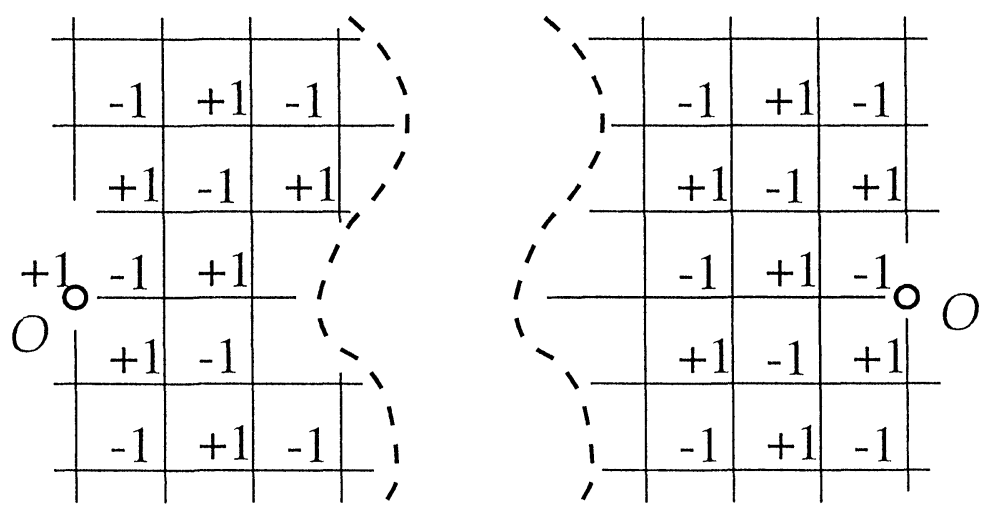

Figure 2

Dobrushin's tour de force was to treat a specification as a primary object, rather than the random field. The question is: how many RFs are there with a given specification? Or, if the specification was constructed as in (16), (17), how many RFs do correspond to a given "potential" $\left\{\Phi_{\Lambda}\right\}$ ? He termed them Gibbs' random fields with a given potential; in the case of the Ising model one speaks of a Gibbs RF with given values of $\beta, \mu$ and $J$. (An alternative term used in the literature is Gibbs' states; we prefer in this article to follow Dobrushin's original terminology.) The uniqueness of a Gibbs RF is treated as the absence of a phase transition and the nonuniqueness as its presence. In this case, it is desirable to describe the structure of the set of RFs with a given specification (reduced to a study of "extremal" or at least translation-invariant "extremal" elements of this set.) ${ }^{3}$ In certain situations (e.g., when the single-spin space $X$ is infinite), the problem of the existence of a Gibbs RF also becomes non-trivial.

At the same time, the paper [56] moved in a similar direction. This explains the term "Dobrushin-Lanford-Ruelle (DLR) state" (or DLR measure).

As noted, Dobrushin produced in [1968b, c] a concise proof of the following fundamental fact.

In the Ising model with zero magnetic field (i.e., $\mu(t) \equiv 0)$, the Gibbs $R F$ is unique when $\beta$ is small, $\beta \leq \beta_{0}$ (i.e., the temperature is high) and non-unique (that is, there are at least two such $R F$ ) when $\beta$ is large, $\beta \geq \beta^{0}$ (i.e., the temperature is low). Furthermore, for $\beta \geq \beta^{0}$ and $J<0$ one of these Gibbs RFs is "close" to the GS $\sigma \equiv+1$ and another to $\sigma \equiv-1$. Similarly, for $\beta \geq \beta^{0}$ and $J>0$ one Gibbs RF is close to the chessboard GS with $\sigma(O)=-1$ and another to that with $\sigma(O)=+1$. Like the corresponding GSs, these RFs are translation-invariant in the ferromagnetic model and translation-periodic in the antiferromagnetic one and are transformed into each other by "flipping" the spins (and also by the unit space shift in the antiferromagnetic case); as $\beta \rightarrow \infty$, they converge to the degenerate probability measures sitting exactly at the corresponding GSs. On the other hand, as $\beta \rightarrow 0$, the (unique) Gibbs' RF converges to the Bernoulli $R F$, with the i.i.d. values $\sigma(t), t \in \mathbb{Z}^{d}$, taking values \pm 1 with probability $1 / 2$.

The proof was based on an ingenious use of the so-called contour technique going back to [75]. The gap between $\beta_{0}$ and $\beta^{0}$ remains wide. It is believed that there exists a "critical" value $\beta_{c r}, \beta_{0}<\beta_{c r}<\beta^{0}$, separating the uniqueness and non-uniqueness regions. In his papers, Dobrushin also gives a general sufficient condition of existence and uniqueness of a Gibbs' RF for a general $X$. We describe Dobrushin's uniqueness condition for the case when $X$ is finite.

\footnotetext{
${ }^{3}$ The picture of the structure of the set of Gibbs' RFs drawn against the values of main parameters (e.g., in the case of the ferromagnetic Ising model, $\beta$ and $\mu$ ) is called the phase diagram.
} 
Let $\alpha(t), t \in \mathbb{Z}^{d}$, be non-negative numbers such that

$$
\sum_{t \neq 0} \alpha(t)<1
$$

Then, if the inequality for the "one-site" conditional probabilities

$$
\begin{gathered}
\frac{1}{2} \sum_{x \in X}\left|P^{\{t\}}\left(\sigma(t)=x \mid \bar{\sigma}_{\mathbb{Z}^{d} \backslash\{t\{}\right)-P^{\{t)}\left(\sigma(t)=x \mid \bar{\sigma}_{\mathbb{Z}^{d} \backslash\{t\}}^{\prime}\right)\right| \\
\leq \sum_{\substack{u \in \mathbb{Z}^{d}: u \neq t\\
}} \alpha(u-t) \mathbf{1}\left(\bar{\sigma}(u) \neq \bar{\sigma}^{\prime}(u)\right)
\end{gathered}
$$

holds true for all $t \in \mathbb{Z}^{d}$ and all configurations $\bar{\sigma}_{\mathbb{Z}^{d} \backslash\{t\}}$ and $\bar{\sigma}_{\mathbb{Z}^{d} \backslash\{t\}}^{\prime}$ on $\mathbb{Z}^{d} \backslash\{t\}$, there exists a
unique Gibbs' $R F$ with the specifications $\left\{P^{\Lambda}\right\}$.

Using this result, he was able to check uniqueness in a large variety of cases, including the ferromagnetic Ising model with $\mu(t) \equiv \mu \neq 0$ and antiferromagnetic with $\mu(t)=-\mu\left(t^{\prime}\right) \neq 0$, $\left\|t-t^{\prime}\right\|=1$, where the GS is unique. He also established uniqueness in a wide class of one-dimensional models (with $d=1$ ) in [1973b] and [1974a, b].

Dobrushin returned to uniqueness problems in [1981a], [1983a], [1985a, b] and [1987a]. In particular, in [1985a, b] and [1990c] a constructive uniqueness criterion was given which required the verification of a finite, though possibly large, number of relations. Under this criterion, the Gibbs' RF had many "nice" properties, which were deemed a "complete analyticity." Here, Dobrushin's earlier idea of contractivity played a crucial role.

In the non-uniqueness direction Dobrushin, following [67], extended the concept of a contour and stated in explicit form the so-called Peierls condition [1974c]. This paper was an important step towards the Pirogov-Sinai theory [77-80] (see also [23]) which gave a powerful method of studying the low-temperature phase diagrams in the absence of symmetry.

One of the most impressive results achieved by Dobrushin in his study of the non-uniqueness was the theorem for the existence of non-translation-invariant Gibbs' RFs for the Ising model with zero magnetic field in dimensions three and above. The point is that, say in the ferromagnetic case, the configuration $\sigma$ given by

$$
\begin{gathered}
\sigma(t)=+1, \text { if } t=\left(t^{1}, \ldots, t^{d}\right) \in \mathbb{Z}^{d} \text { and } t^{d} \geq 0, \\
=-1, \text { if } t=\left(t^{1}, \ldots, t^{d}\right) \in \mathbb{Z}^{d} \text { and } t^{d}<0
\end{gathered}
$$

(see Figure 3) is also a GS, according to a "reasonable" formal definition. The question is whether the configuration $\sigma$ in (21) "generates" a Gibbs RF in a way similar to the above translationinvariant GSs. In [1973a] Dobrushin gave a positive answer for $d \geq 3$ and $\beta$ large enough, $\beta \geq \beta^{1} \gg \beta^{0}$. Furthermore, as he believed, his results suggest that in dimension $d \geq 3$ there should be another critical value $\beta_{c r}^{1}>\beta_{c r}$ such that the non-translation-invariant Gibbs RFs appear for $\beta>\beta_{c r}^{1}$ but not for $\beta_{c r}<\beta<\beta_{c r}^{1}$. For the two-dimensional Ising model $(d=2)$ the answer to the above question is negative, and for all $\beta>\beta_{c r}$ there are only two extremal Gibbs RFs [1, 40]. Dobrushin came to a similar conclusion assuming that $\beta$ large enough, but in a wider class of two-dimensional models [1985e].

Apart from the above series of results, Dobrushin progressed in a variety of other directions in equilibrium statistical mechanics and related areas of probability theory. We give below a far from exhaustive list of his activities. 


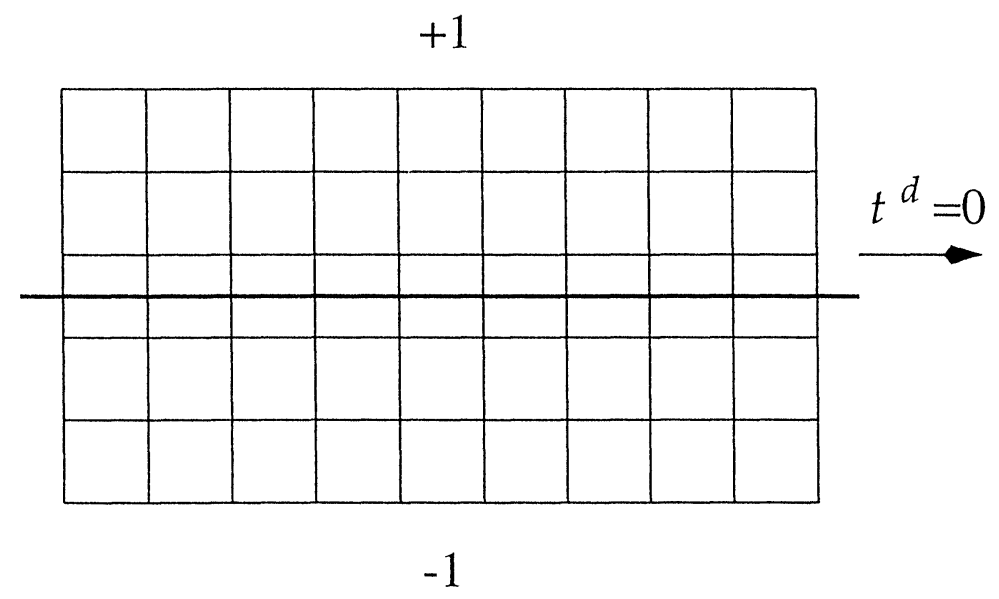

Figure 3

He analyzed logarithmic asymptotics of the partition function and their relation to the phase diagram [1967a], [1972a], [1974d]. He also contributed to the study of the problem of equivalence of ensembles [1977a].

He studied models with a continuous spin space $X$, producing spectacular results both for the absence of phase transitions [1975a], [1978b] and their presence [1981b], [1986a]. He also considered models with "non-standard" interaction potentials $[1988 \mathrm{~b}, \mathrm{c}]$ showing unusual features of phase transitions.

Investigating into the behavior at a critical point, he shaped the theory of auto-model (selfsimilar) RFs. In particular, he found a new class of Gaussian auto-model (or self-similar) RFs [1978a], [1979a], [1981c], [1988a].

In connection with the Euclidean quantum field theory, he established various properties of Gaussian RFs [1979b, e], [1980a], [1983c, d]. We also mention an attempt to study the Euclidean phase diagram of the two-dimensional boson $P(\phi)_{2}$ model. This attempt was unfortunately not completed (after an announcement [1973c] there was no detailed proof published, and the credit justifiably was transferred to [26-29] and [19] (see also [25])), but the by-products of these studies filled the papers [1975c], [1976a], [1978d] and provided a score of new results from the theory of generalized Markov RFs on $\mathbb{R}^{d}$.

A striking example of Dobrushin's creative ideas is connected with the problem of describing specifications in terms of a potential. The question is whether the system conditional probabilities of a Markov or "approximately Markov" RF can be written in a "Gibbsian" form (14)-(16) for a suitable potential. In a sense, this may be regarded as an "inverse" problem to problem of phase transition. The answer is yes, at least under "natural" regularity conditions on the specification. See [5-7, 31, 51-52, 90, 95] and [99]. Dobrushin himself did not publish any result in this direction, but his ideas were used in most of the related publications.

In the late eighties, Dobrushin began, following earlier works $[68,69]$, a detailed study of the geometry of random shapes that separate different phases in the plane Ising model. Heuristically, the way of describing such a shape was discussed in the last century by the Russian physicist Wulff [101] (who proposed a rather general theory of shapes of surface tension). The rigorous form of his theory for the two-dimensional Ising model was provided in the monumental papers $[1989 \mathrm{~b}],[1992 \mathrm{a}, \mathrm{b}]$ continued in [1993d], [1994c], [1995a, b] and [1996b]. The extension of this approach to the Ising model in higher dimensions remains an open challenging problem. 
As a by-product of his studies in the Wulff theory in the late eighties, Dobrushin became deeply interested in the large deviations approach to the theory of phase transitions [1992c], [1994c, d]. Dobrushin's idea was that the famous Cramer transformation playing the crucial role in the analysis of the large deviation probabilities is closely connected to the Gibbs representation (16). In fact, he believed in a universality of the large deviations technique and tried to use it in various areas, including queueing network theory, see below.

\subsection{Non-equilibrium statistical mechanics and processes with local interaction}

At the end of the sixties, Dobrushin showed considerable interest in problems arising in the theory of random automata networks. Initial impetus for research in this direction was given in the sixties by Pyateckii-Shapiro and his co-workers who actively discussed related problems at a seminar series at Mekh-Mat. In Dobrushin's interpretation, with each site $x$ of a lattice $\mathbb{Z}^{d}$, one associates a random process $\eta^{x}$, with discrete or continuous time, and the conditional probability $\pi^{x}\left(s \mid\left\{s_{y}\right\}\right)$ for process $\eta^{x}$ to be at time $t+1$ in state $s$ given the states $s_{y}$ of processes $\eta^{y}, y \in \mathbb{Z}^{d}$, at the preceding time is determined by the $s_{y}$ 's with $\|y-x\| \leq R_{0}$ where $R_{0}$ is a constant. (In the continuous-time set-up, one has in mind the conditional rate of the jump of $\eta^{x}$ from $s_{x}$ to $s$.) Furthermore, the different processes $\eta^{x}$ evolve (conditionally) independently. One can say that the whole family $\left\{\eta^{x}, x \in \mathbb{Z}^{d}\right\}$ forms a Markov process $\eta$ (with a continual state-space). Such processes were later called Markov processes with local interaction. In [1971a, b], Dobrushin gave a formal construction of such a process and established sufficient conditions for convergence to an invariant distribution. He also showed that the reversible invariant distributions are precisely Gibbs RFs on $\mathbb{Z}^{d}$ with a potential that is naturally calculated in terms of the conditional probabilities $\left\{\pi^{x}\right\}$. His papers are considered the origin of a theory that later became a well-established field of probabilistic research, see [59].

Another direction of Dobrushin's interests was the construction of the so-called non-equilibrium dynamical systems of statistical mechanics. The problem is as follows. Consider a Hamiltonian system of equations in the $d$-dimensional Euclidean space

$$
\frac{d}{d t} q_{j}(t)=p_{j}(t), \quad \frac{d}{d t} p_{j}(t)=-\operatorname{grad} \sum_{k: k \neq j} V\left(\left\|q_{j}(t)-q_{k}(t)\right\|\right),
$$

with the Hamiltonian

$$
H\left(\left\{q_{j}, p_{j}\right\}\right)=\frac{1}{2} \sum_{j}\left(p_{j}^{2}+\sum_{k: k \neq j} V\left(\left\|q_{j}-q_{k}\right\|\right)\right) .
$$

Here $q_{j}, p_{j} \in \mathbb{R}^{d}$ are the positions and momenta of particles and $V$ the pair interaction potential depending on the Euclidean distance (the mass of a particle is taken to be one). A typical shape of $V(r), r \geq 0$, is given in Figure 4 below (the so-called Lennard-Jones type potential). The value $a \geq 0$ is the "hard core" diameter. The behavior of $V$ for $r \sim a$ reflects the repulsion when particles are near each other while decreasing as $r \rightarrow \infty$ the decay of the interaction at large distances. For $r>a, V(r)$ is supposed to be smooth in $r$.

One is interested in solving the Cauchy problem for (22), with initial data (ID)

$$
q_{j}(0)=q_{j}^{0}, p_{j}(0)=p_{j}^{0} \text {. }
$$

If the number of particles is finite, the solution of (22), (24) exists and is unique for a "massive" set of ID $\left\{q_{j}^{0}, p_{j}^{0}\right\}$ (exceptional cases are where $\left\|q_{j}^{0}-q_{k}^{0}\right\|<a$ for some $j \neq k$ and, depending on the shape of $V$ for $r \sim a$, some other "singular" ID (e.g., leading to triple collisions)). See, e.g., [76]. A similar assertion also holds where the particles are confined to a bounded domain $\mathbb{D} \subset \mathbb{R}^{d}$, with the boundary condition of elastic reflection (or put on the $d$-dimensional torus $\mathbb{T}^{d}$ ). However, in statistical mechanics, one considers the limit with an infinite system of type (22). Hamiltonian (23) then becomes, as a rule, a formal expression, and the traditional way of proving the existence and uniqueness of the solution of the Cauchy problem (22), (24) fails. In fact, it is 
possible to construct examples of ID for which the solution "blows" up or is not unique.

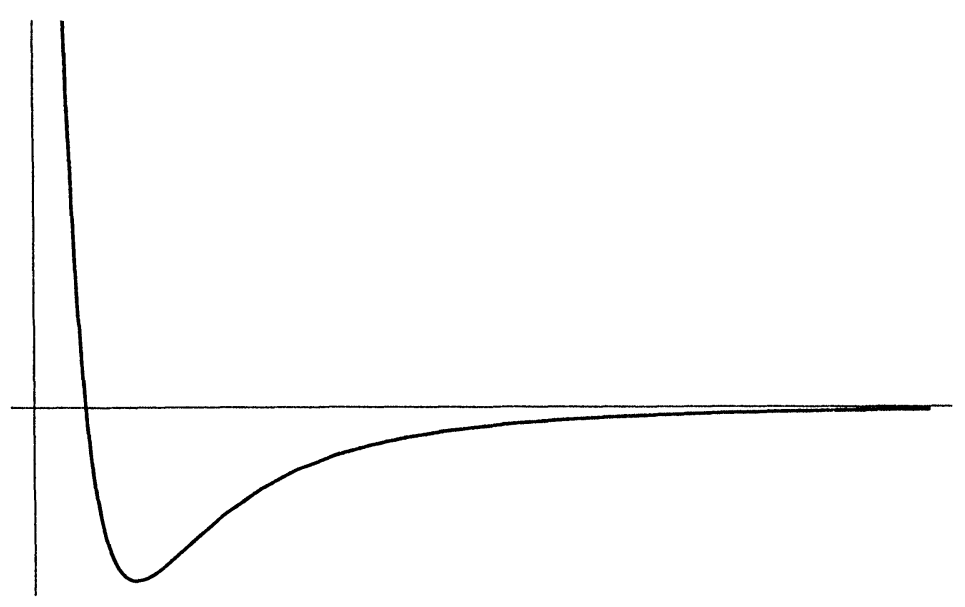

Figure 4

However, the following remark saves the day. For "large" systems, one is interested not in the evolution of an "individual" ID, but rather of a probability distribution. That is, one is concerned with having a solution to (22), (24) not for any ID, but for the set of ID supporting a "natural" probability distribution.

First results in this direction were obtained in $[53,54]$ and (for a different class of systems) in [55]. In particular, in [53,54] the one-dimensional case $d=1$ was considered, with a potential $V$ that was of a finite range $\left(V(r)=0\right.$ for $\left.r>R_{0}\right)$ and without hard core (i.e., with no singularity for $0 \leq r \leq \infty)$. The result was that the existence and uniqueness of a solution of an infinite system (22) hold for a "massive" set of ID which have probability one with respect to a large class of measures on the phase space of infinitely many particles. This set was described in asymptotical terms, as well as the class within which existence and uniqueness hold.

The above restrictions on $d$ and $V$ were not considered to be natural, and many researchers tried to remove them. An alternative approach was proposed in [93] and extended in a series of papers completed with [81]. Here, dimension $d$ was ultimately made arbitrary and the condition on $V$ allowed to include the potential of the type in Figure 4. However, the price to pay was that the set of ID was made "less massive." One could only guarantee that it had probability one with respect to any Gibbs RF with potential $V$. Such a random field is defined and constructed in a similar fashion to the lattice case discussed in Section 2.3. It turns out to be invariant (or equilibrium) probability distribution under the shift along the solutions of (22). Owing to this fact, results of this kind were referred to as equilibrium dynamical systems. In [1977b, c] Dobrushin proposed a new construction of the solution to (22), (24) which allowed him to include, in dimensions $d=1$ and 2, "realistic" potentials $V$ and establish the existence and uniqueness for a set of ID having probability one with respect to a large class of measures, not necessarily equilibrium ones. Up to now, these results have not yet been improved upon. In particular, Dobrushin conjectured that in dimension $d \geq 3$ the problem of finding a "good" set of ID has a negative answer.

After constructing a dynamical system of infinitely many particles, one naturally asks whether it has "ergodic" properties of one kind or another. Dobrushin [1978c], [1985f], [1993b] believed that "typically" such systems should exhibit convergence to a limiting distribution at 
large times, and the limit has to be a Gibbs RF with the potential $V$ figuring in the original system (22). He even produced a physical picture of such convergence. Formally, however, he was able to check this fact only for some "degenerate" models [1979d]. We give here the corresponding result for the so-called one-dimensional system of hard-rods. Equations of motion may be formally written in the form (22), with $d=1$ and the potential $V(r)$ taking values 0 and $\infty$ depending on whether $r>a$ or $r \leq a$ where $a>0$ is the diameter of a hard rod. See Figure 5 below.

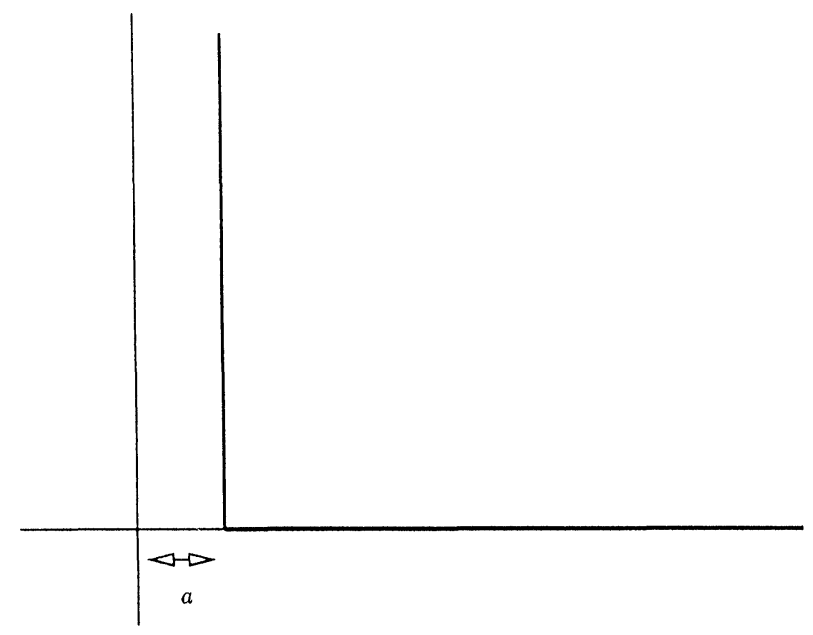

Figure 5

Pictorially, the particles move on line $\mathbb{R}^{1}$ freely when they are apart (i.e., $q_{j+1}-q_{j}>a$ ). When they collide (i.e., $q_{j+1}-q_{j}=a$ ) they exchange their momenta. Such a system may be considered completely integrable. The number (or fraction) of particles with a given momentum is preserved in time. Dealing with hard rods, it is convenient to think of a "contraction" that reduces a hard rod to a point particle. The motion of the rods is then transformed into free motion. Conversely, a "dilation" map transforms free motion into that of hard rods.

An equilibrium (invariant) distribution $\mathbb{P}$ for an infinite hard-rod system is a random marked point process on the line $\mathbb{R}^{1}$ with marks (momenta) from $\mathbb{R}^{1}$ determined by the following conditions:

(i) the distribution of the positions $\left\{q_{j}\right\}$ is translation-invariant, and, given that the origin is "covered" by hard rod (i.e., $\left|q_{i}\right| \leq a / 2$ for some $i$ ), the distances $q_{j+1}-q_{j}-a$ between subsequent pairs of rods are (conditionally) i.i.d. and have an exponential distribution of mean $\lambda^{-1}$;

(ii) the momenta of the particles are i.i.d. random variables; their common distribution is denoted by $\nu$.

The particle density under distribution $\mathbb{P}$ equals $\rho=\frac{\lambda}{1+a \lambda}$. The above contraction and dilation maps take this distribution to a Poisson marked process of rate $\lambda$ with i.i.d. marks and vice versa.

As was proved in [2] and [92], the equilibrium dynamical system with an invariant measure of the above type has (depending on $\nu$ ) good ergodic properties. Dobrushin extended such a picture to a wider class of non-invariant measures. Namely:

Suppose $\mathbb{Q}$ is an arbitrary translation-invariant marked point process of density $\rho$ and with an individual momentum distribution $\nu$. Assume $\mathbb{Q}$ satisfies a condition of space mixing (see $[1979 \mathrm{~d}])$. Then the process $\mathbb{Q}_{t}$ obtained from $\mathbb{Q}$ in the course of the hard-rod dynamics converges as $t \rightarrow \infty$ to the equilibrium distributions $\mathbb{P}$ with the same density $\rho$ and momentum distribution $\nu$. 
The proof of this result was based on the aforementioned connection with the free motion for which a similar convergence was established in [1979d], [1980e] in a wide situation (including any dimension $d \geq 1$ ). As noted in Section 2.1, the main ideas here go back to [1956e].

Continuing further in this direction, Dobrushin pioneered the study of the so-called hydrodynamical limit (HL). This idea goes back to an earlier paper [71] (in the physical literature, similar attempts may be traced to the thirties and forties. See e.g., [16] and references therein). The problem is to establish a formal connection between system (22) describing the motion of particles (i.e., dynamics at the micro-level) and hydrodynamical equations (Euler and NavierStokes) describing the "collective" motion of a fluid or dense gas medium (i.e., a dynamics at a macro-level). The existing ways of deriving Euler and Navier-Stokes equations are essentially heuristic; to various degrees the same is true of other so-called kinetic equations (except for the Vlasov equation which Dobrushin exhaustively studied in [1979c]). For details, see [97]. The attempt to derive the Euler equation in [71] was made under certain formal assumptions about the solutions of the so-called BBGKY hierarchy equations. A careful analysis of these assumptions shows that they are related to delicate ergodic properties of system (22) which up to now have not been verified (and may not hold). Dobrushin adopted a different point of view. He attempted to perform the HL for special models where assumptions from [71] (or their equivalent) may be verified and the HL performed in a formally correct fashion. These models, though nontrivial, may be too idealistic to lead to the usual Euler or Navier-Stokes equations (Dobrushin used the term "caricatures of hydrodynamics"), but they display a mechanism behind the HL which is believed to hold in more realistic systems. Dobrushin's results for such caricature models were published in [1980b-d], [1982a, b], [1983b], [1985f], [1986c], [1988e], [1989a], [1990b], [1991a] and $[1993 \mathrm{~b}]$.

It has to be said that Dobrushin's papers [1980b-d], [1982a, b] and [1983b] were the first ones to contain together the mathematically correct definition and the rigorous proof of the HL in the form that is commonly used since then in the modern literature. (The definition of the HL was independently proposed in [96] on the basis of physical considerations.) The main feature of the HL is the space-time scaling. In physical terms, one considers a family of probability distributions $\left\{\mathbb{P}^{\epsilon}\right\}$ of a particle systems which changes in space on the scale $\epsilon^{-1}$. This means that the average parameters of interest calculated around the space point $q=\epsilon^{-1} x$ under $\mathbb{P}^{\epsilon}$ are nice functions of $x \in \mathbb{R}^{d}$. In [71] such parameters were the particle density $\rho(x)$, the density of momentum $\mathbf{p}(x)=$ $\left(p_{1}(x), \ldots, p_{d}(x)\right)$ and the density of energy $e(x)$. One then performs the shift of $\mathbb{P}^{\epsilon}$ along the solutions of (22) by time $t=\epsilon^{-1} \tau, \tau \in \mathbb{R}^{1}$, and calculates, in the shifted distribution $\mathbb{P}_{\epsilon}^{\epsilon}-1_{\tau}$, the above quantities, obtaining functions $\rho(\tau ; x), \mathbf{p}(\tau ; x)=\left(p_{1}(\tau ; x), \ldots, p_{d}(\tau ; x)\right)$ and $e(\tau ; x)$ (more precisely, these functions arise as the limits as $\epsilon \rightarrow 0)$. Under the assumptions that have been made in [71], these functions satisfy the Euler equation for a compressible fluid

$$
\begin{gathered}
\frac{\partial}{\partial \tau} \rho(\tau ; x)=-\operatorname{div}[\rho \mathbf{p}] \\
\frac{\partial}{\partial \tau}\left[\rho p_{i}\right](\tau ; x)=-\operatorname{div}\left[\rho p_{i} \mathbf{p}\right](\tau ; x)-\frac{\partial}{\partial x_{i}} \mathscr{P}(\tau ; x), i=1, \ldots, d, \\
\frac{\partial}{\partial \tau}[\rho e](\tau ; x)=-\operatorname{div}[\rho e \mathbf{p}-P \mathbf{p}](\tau ; x) .
\end{gathered}
$$

Parameters $x$ and $\tau$ are related here to the macro- whereas $q$ and $t$ are related to the micro-scale. The quantity $\mathscr{P}$ is a function of $\rho, \mathbf{p}$ and $e$ giving the pressure of the system with interaction potential $V$ from (22). It is related to the logarithmic asymptotics of the partition function with given values of the particle number, momentum and energy densities. The appearance of the functions $\rho, \mathbf{p}$ and $e$ are not occasional. These functions give the space-time densities of the fundamental conserved quantities of motion: the number of particles, the total momentum and the total energy. As was shown in [36-39] and [33], for a "generic" potential $V$, the above "canonical" first integrals are the only possible invariants of the motion of an infinite system which satisfy a 
natural additivity condition; it is this condition that allows one to use them in equation (25) and alike. On the other hand, there exist "exceptional" potentials for which the family of additive invariants of the motion includes "exotic" first integrals. In dimension one $(d=1)$ these potentials have been investigated in [34]. The hard-rod potential on Figure 5 is one of them.

Correspondingly, the one-dimensional hard-rod system was one of the first caricature models to be investigated in connection with the HL. See [1980c, d] and [1983b]. As already observed, in this model there exists an abundance of the constants of motion. Instead of a triple $(\rho(\tau ; x), \mathbf{p}(\tau ; x), e(\tau ; x))$ one has to deal here with density $\varrho(\tau ; x, v)$ of the particles with momentum $v$ at (macro-) point $x$ at (macro-) time $\tau$. The above scheme can then be carried through and the following quasilinear hyperbolic partial differential equation emerges in the HL:

$$
\begin{aligned}
& \frac{\partial}{\partial \tau} \varrho(\tau ; x, v) \\
& =\frac{\partial}{\partial x}\left[\left(-v+\int d v^{\prime}\left(v-v^{\prime}\right) \varrho\left(\tau ; x, v^{\prime}\right)\left(1-a \int d w \varrho(\tau ; x, w)\right)^{-1}\right) \varrho(\tau ; x, v)\right] .
\end{aligned}
$$

If the initial function $\varrho(0 ; \cdot, \cdot) \geq 0$ obeys $\sup _{x \in \mathbb{R}^{1}} \int d w \varrho(0 ; x, w)<a$ and $\sup _{x \in \mathbb{R}^{1}} \int d w|w| \rho$ $(0 ; x, w)<\infty$ then the solution of $(26)$ exists, is unique for all $\tau \in \mathbb{R}^{1}$, and satisfies the same conditions.

Equation (26) may be considered as an analog of the Euler equation for a hard-rod "fluid." The hard-rod model remains the only example of a "nonlinear" Hamiltonian system with interaction where the HL was performed at a rigorous level with no additional assumption. Recently, the standard Euler equation was derived in [73] in the situation where equations of motion include "stochastic" terms which remove the main difficulties one had to contend with in [71].

Dobrushin also spent a considerable time in thinking of how the Navier-Stokes equation should be related to the HL procedure. His point of view was that it arises when one takes into account the "next" correction to the limiting Euler equation, up to the order $\varepsilon$. Such an approach was not unanimously approved among the specialists, but confirmed on caricature models [1982b], [1988e] and [1990b].

A separate (although close) direction is the HL for various stochastic models, including processes with local interactions. Dobrushin's ideas inspired many works in this field. His own results in this direction are published in [1982b] and [1991a].

\subsection{Queueing network theory}

The last field of Dobrushin's research on which we are going to comment is queueing network (QN) theory. As was mentioned above, he was driven by fruitful analogies between this theory and several areas of statistical physics. In both fields, one deals with a large system characterized by complex interaction between its "components." It must be noted that at the beginning of the seventies when he began working in this direction, queueing theory was essentially oriented towards problems related to an isolated device, with one or several channels of service. Under certain independence (and sometimes exponentiality) assumptions, an array of elegant formulas was produced for the distribution and expected values of various random variables characterizing such a queue, i.e, waiting time, queue size (number of customers in the queue), duration of a busy or idle period, etc. Below we use the term the Pollaczek-Khintchine-type formulas when referring to results of this kind, see, e.g. [32].

In $\mathrm{QN}$ theory, there existed papers [41, 42] forming a particular approach to QN problems (the term Jackson's networks was coined for the network class considered in these papers). The results of $[41,42]$ demonstrated striking features of the coming theory, but the consensus was that in general, QNs are too complicated to be successfully studied at a mathematically rigorous level. 
The class of Jackson's networks was later considerably extended [49, 50], but even the new class (afterwards called Kelly's networks) was quite restrictive for many applications.

Dobrushin was not deterred by the apparent complexity of QNs. On the other hand, he was not happy with the rather special exponentiality and independence assumptions made in the above papers leading to the so-called product-form of the invariant distribution in Jackson's and Kelly's networks. This was perhaps partly because of his general reservations about exactly solvable models. First, he proposed the so-called mean-field approach to the QN theory which he demonstrated in [1976b] on the example of a so-called star-shaped message-switched network.

Such a network consists of a center $C$ and a number of input and destination nodes (senders and receivers, respectively), $S_{1}, \ldots, S_{M}$ and $D_{1}, \ldots, D_{N}$, connected by the directed lines as on Figure 6 below.

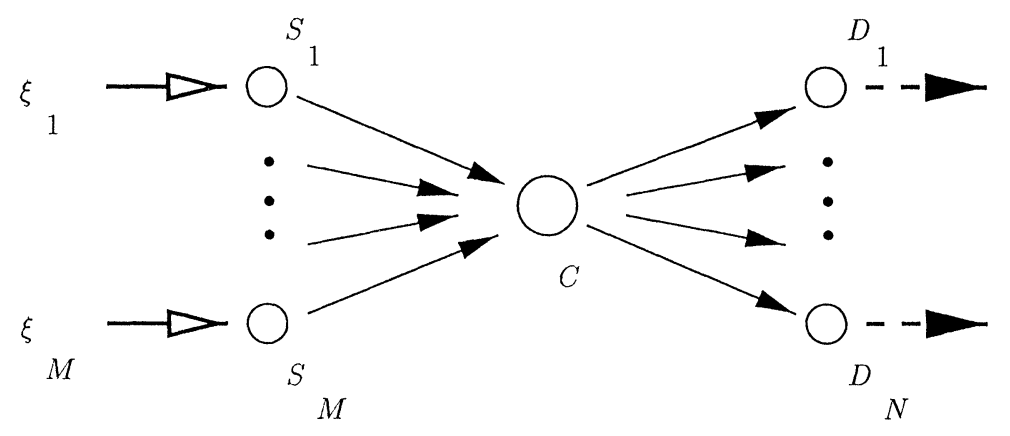

Figure 6

On the input port of each line $S_{i} \rightarrow C$ and $C \rightarrow D_{j}$, there is a single server that processes (or transmits along the line), on the FCFS basis, a corresponding stream of arriving "messages." Messages originally appear in nodes $S_{1}, \ldots, S_{M}$ (or at the input ports of lines $S_{i} \rightarrow C, 1 \leq i \leq M$ ); one assumes for definiteness that their arrival there is described by i.i.d. Poisson marked processes $\xi_{1}, \ldots, \xi_{M}$ of rate $\lambda$, each with i.i.d. marks. The marks in each process $\xi_{i}$ are triples $\left(b ; l^{1}, l^{2}\right)$ where $b=1, \ldots, N$ is the address of a message and $\left(l^{1}, l^{2}\right)$ the pair of its service (or transmission) times along lines $S_{i} \rightarrow C$ and $C \rightarrow D_{b}$, respectively. The "individual" mark distribution is as follows:

(a) the $b$ 's are equi-distributed $(\mathbb{P}(b=j)=1 / N)$;

(b) the pair $\left(l^{1}, l^{2}\right)$ is independent of $b$ and has a fixed joint distribution $\nu$ (e.ge, $l^{1}$ and $l^{2}$ may be independent or coincide $\left.\left(l^{1}=l^{2}\right)\right)$.

After being processed along the line $S_{i} \rightarrow C$, a message from $\xi_{i}$ with address $b=j$ immediately joins the queue for the line $C \rightarrow D_{j}$. After being processed along this line, it is considered delivered to its destination node $D_{j}$ and disappears from the network. One is interested in the distribution of the end-to-end delay of a given message, i.e., the time from a message appearance in node $S_{i}$ to its delivery at $D_{j}$. This is clearly the sum $w^{1}+w^{2}+l^{1}+l^{2}$ where $w^{1}$ and $w^{2}$ are message's waiting times for server $S_{i} \rightarrow C$ and server $C \rightarrow D_{j}$, respectively. To formally define the corresponding random variables, we use the so-called Palm distribution where one of the messages is "tagged" and followed through its journey along the path $S_{i} \rightarrow C \rightarrow D_{j}$. Assume that $[M / N]$ is keep fixed an equal to $\kappa$, and the non-overload conditions

$$
\frac{\lambda}{\mu^{1}}, \frac{\lambda}{\mu^{2}}<1
$$

hold where $\mu^{1}=\left(\int \nu\left(d l^{1} \times d l^{2}\right) l^{1}\right)^{-1}, \mu^{2}=\left(\kappa \int \nu\left(d l^{1} \times d l^{2}\right) l^{2}\right)^{-1}$. The distribution of $w^{1}$ is then given by a well-known Pollaczek-Khinchtine-type formula; for example, if $l^{1}$ has the exponential 
distribution of rate $\mu^{1}$,

$$
\begin{gathered}
\mathbb{P}\left(w^{1}>x\right)=\frac{\lambda}{\mu^{1}} \exp \left[\left(\lambda-\mu^{1}\right) x\right], x \geq 0, \\
=1, x<0 .
\end{gathered}
$$

However, the joint distribution of $w^{1}, w^{2}$ and $\left(l^{1}, l^{2}\right)$ (and even the marginal distribution of $w^{2}$ ) cannot be written in a closed form. The theorem (in a slightly more general form than that given in $[1976 \mathrm{~b}])$ is as follows.

Suppose that $M, N \rightarrow \infty$. Then the limiting distribution of random variable $w^{2}$ is as in (28), replacing $\mu^{1}$ with $\mu^{2}$. Furthermore, random variables $w^{1}$ and $w^{2}$ become asymptotically independent and independent of the pair $\left(l^{1}, l^{2}\right)$. Hence, the limiting distribution of the end-to-end delay is the convolution of those of $w^{1}$ and $w^{2}$ and $l^{1}+l^{2}$.

This result means that the network with large values of $M, N$ operates as a collection of nearly independent servers each of which has to process a stream of tasks close in distribution to a point process of a simple form (in the example under consideration, to a Poisson process). The analogy with the mean-field picture in statistical mechanics is that each queue in the network becomes asymptotically independent of the rest. However, the influence of the whole network on a given server is manifested through the form of an "averaged" input stream feeding this server. The mean-field approach proved to be very rewarding and was later developed in a number of works. See, e.g., the reviews [48] and [1990a] and the references therein, as well as the paper [43]. Dobrushin returned to the mean-field-type results in his latest publication [1996a] where he studied an example of a network with elements of control.

The network under consideration in [1996a] is pictured in Figure 7 below.

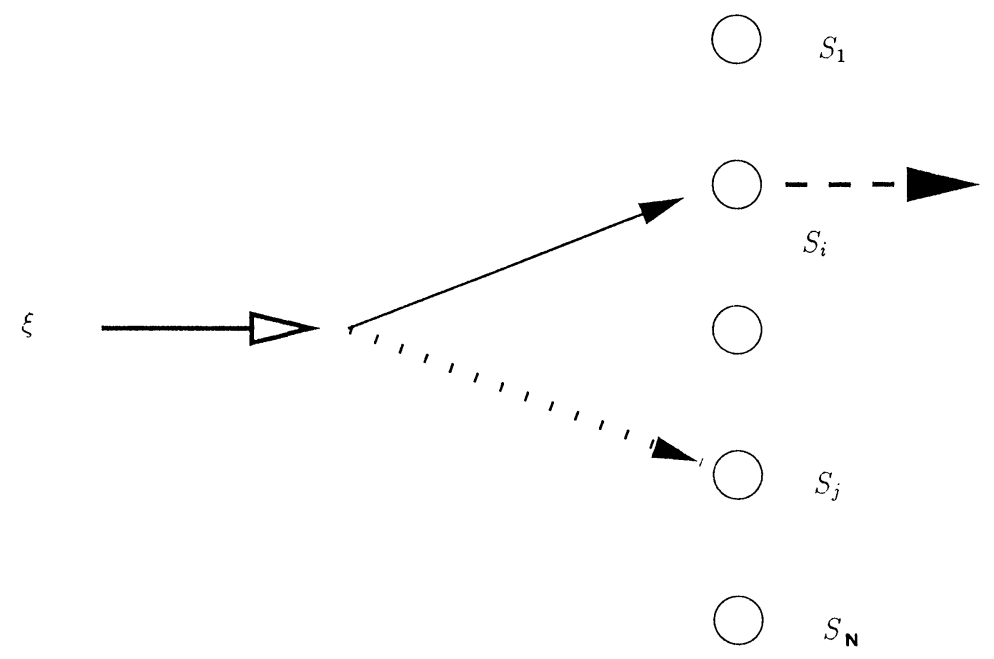

Figure 7

It contains $N$ single servers $S_{1}, \ldots, S_{N}$ fed with common exogenous stream of tasks $\xi$ which is assumed to be Poisson of rate $\lambda N$. Let the service times of the task be i.i.d., with the exponential distribution of mean $\mu^{-1}$ and suppose the non-overload condition

$$
\frac{\lambda}{\mu}<1
$$

to be valid. Assume that, at the time of task's arrival, one picks randomly a pair of servers, $S_{i}$ and $S_{j}$, and then selects the one of the two with the shorter queue. One is interested in the distribution of the queue size per server in such a network. It is clear that this distribution must be "better" than if the servers were selected completely randomly. The latter model can be solved 
by means of the Pollaczek-Khintchine-type formulas.

It was proved in [1996a] that:

As $N \rightarrow \infty$, the average queue size distribution in the network with the above selection rule has the limiting distribution

$$
\mathbb{P}(Q \geq m)=\left(\frac{\lambda}{\mu}\right)^{2 m-1}, \quad m \geq 1 .
$$

In the case of the completely random choice the probability in (30) equals $\left(\frac{\lambda}{\mu}\right)^{m}$ and therefore decays much slower with $m$. This illustrates the benefits of the control introduced in the model.

Another direction initiated by Dobrushin in the QN theory was focused on the concept of an infinite network. The aim here was to grasp another feature on the behavior of complex QN's, instability of a stationary regime. Formally, it should be manifested in non-uniqueness of an invariant distribution in a network with infinitely many nodes as opposite to the uniqueness for its finite counterpart. This is a striking analogy with the theory of phase transitions. In [1979f], Dobrushin has made an initial step in this direction, proving that for message-switched networks on the infinite one-dimensional lattice the concept of an invariant distribution may be correctly defined, and in the situation of small "transit" flows such a distribution is unique. This results influenced a series of subsequent papers, see e.g., [11], [46, 47], and in particular [45], where Jackson's networks on infinite graphs were analyzed and a non-uniqueness of an invariant distribution has been established.

The third direction in the QN theory connected with the Dobrushin's name was concerned with general non-overload conditions guaranteeing the existence of a stationary regime. [In analogy with his information-theoretical studies, he used the term the network capacity region.] The problem here is to determine conditions in terms of expected values, similar to (27), (29), under which the queues in a given network do not "blow-up." For Jackson's and Kelly's networks, these conditions may be directly derived from the product-form of the invariant distribution. Dobrushin believed that similar conditions hold for a general class of networks, but his conjecture was later disproved (see, e.g, $[17,18]$ ). He has not published any result in this direction, but his ideas were instrumental for a number of papers (see again, the reviews [48] and [1990a] and a recent work [87]). He also participated in the analysis of the form of an invariant distribution for a general QN. More precisely, he associated with a network an "input-output transformation" that takes an exogenous flow entering the network to the departure flow that leaves it. Many of his predictions about the existence of and convergence to an invariant distribution for such a transformation turned out to be correct, after recent works [4], [72] and [100].

As noted, in the late eighties, Dobrushin started an active research of a large deviations approach to various problems, in particular, in QN theory. His results are contained in [1994b, e]. In particular, in [1994b] he analyzed the probability of a large deviation for the waiting time in a tandem single-server network. He discovered the so-called bottleneck phenomenon that the logarithmic asymptotics of this probability is determined by the "slowest" server. The proof is based on an elegant representation of the waiting time in terms of the input flow. This allowed him to consider wide classes of exogenous processes, in contrast with most of the papers in the field where one has to introduce rather restrictive exponentiality assumptions.

\section{Dobrushin's List of Publications}

\section{2}

a) On regularity conditions for time-homogeneous Markov processes with a countable set of possible states, Uspekhi Matematicheskih Nauk 7:6 (1952), 185-191. [Russian]

1953

a) A generalization of the Kolmogorov equations for Markov processes with a finite number of 
possible states, Matematicheskii Sbornik 33:3 (1953), 567-596. [Russian]

b) A limit theorem for Markov chains with two states, Izvestia Akademii Nauk SSSR, Seriya Matematich. 17:3 (1953), 291-330. [Russian] Translated in: Select. Transl. Math. Stat. and Prob. 1 (1961), 97-156.

\section{4}

a) Conditions of regularity for Markov processes with a finite number of possible states, Matematich. Sbornik 34:3 (1954), 541-556. [Russian]

\section{5}

a) A lemma on the limit of a superposition of random functions, Uspekhi Matematicheskih Nauk 10:2 (1955), 157-159. [Russian]

b) Two limit theorems for the simplest random walk on a line, Uspekhi Matematicheskih Nauk 10:3 (1955), 139-146. [Russian]

c) Central limit theorem for non-stationary Markov chains, Doklady AN SSSR 102:1 (1955), 5-8. [Russian]

\section{6}

a) On conditions for the central limit theorem for non-stationary Markov chains, Doklady AN SSSR 108:6 (1956), 1004-1006. [Russian]

b) Central limit theorem for non-stationary Markov chains I, Teoriya Veroyat. $i$ ee Primeneniya 1:1 (1956), 72-89. [Russian] Translated in: Theory Prob. Its Appl. 1 (1956), 65-80.

c) Central limit theorem for non-stationary Markov chains II, Teoriya Veroyat. $i$ ee Primeneniya 1:4 (1956), 365-425. [Russian] Translated in: Theory Prob. Its Appl. 1 (1956), 329-383.

d) An example of a countable homogeneous Markov process all states of which are transient, Teoriya Veroyat. $i$ ee Primeneniya 1:4 (1956), 481-485. [Russian] Translated in: Theory of Prob. Its Appl. 1 (1956), 436-440.

e) On Poisson laws for the distributions of particles in space, Ukrainskii Mathematicheskii Zhurnal 8:2 (1956), 127-134. [Russian]

f) (With A.M. Jaglom), A complement of the translators, In: J. Doob. Stokhasticheskie Protsessy. Izdatel'stvo Inostrannoii Literatury, Moscow (1956), 576-688. [Russian] (A complement to the Russian translation of: Doob, J.L., Stochastic Processes, Wiley, New York 1953).

\section{7}

a) Some classes of homogeneous denumerable Markov processes, Teoriya Veroyat. $i$ ee Primene niya 2:3 (1957), 377-380. [Russian] Translated in: Theory Prob. Its Appl. 2 (1957), 366-369.

\section{8}

a) The continuity condition of a sample function of a martingale, Teoriya Veroyat. $i$ ee Primeneniya 3:1 (1958), 97-98. [Russian] Translated in: Theory Prob. Its Appl. 3 (1958), 92-93.

b) A statistical problem arising in the theory of detection of signals in the presence of noise in a multichannel system and leading to stable distribution laws, Teoriya Veroyat. $i$ ee Primeneniya 3:2 (1958), 173-185. [Russian] Translated in: Theory Prob. Its Appl. 3 (1958), 161-173.

c) Information transmission in a channel with feedback, Teoriya Veroyat. i ee Primeneniya 3:4 (1958), 395-412. [Russian] Translated in: Theory of Prob. Its Appl. 3 (1958), 367-383.

d) A simplified method of experimentally evaluating the entropy of a stationary sequence, Teoriya Veroyat. i ee Primeneniya 3:4 (1958), 462-464. [Russian] Translated in: Theory Prob. Its Appl. 3 (1958), 428-430.

\section{9}

a) A general formulation of the basic Shannon theorem in information theory, Doklady AN SSSR 126:3 (1959), 474-477. [Russian]

b) A general formulation of the basic Shannon theorem in information theory, Uspekhi Matematicheskih Nauk 14:6 (1959), 3-104. [Russian] Translated in: AMS Translations 33 Series 2 (1961), 323-438. 
c) Optimum information transmission through a channel with unknown parameters, Radiotehnika $i$ Electronika 4:12 (1959), 1951-1956. [Russian] Translated in: Radio Engineering and Electronics 4:12 (1959), 1-8.

\section{0}

a) Passage to the limit under the information and entropy signs, Teoriya Veroyat. $i$ ee Primeneniya 5:1 (1960), 29-37. [Russian] Translated in: Theory Prob. Its Appl. 5 (1960), 25-32.

b) Properties of sample functions of a stationary Gaussian process, Teoriya Veroyat. i ee Primeneniya 5:1 (1960), 132-134. [Russian] Translated in: Theory Prob. Its Appl. 5 (1960), 120-122.

c) The asymptotic behavior of the probability of errors when information is transmitted through channel without memory with symmetric matrix of transition probabilities, Doklady AN SSSR 133:2 (1960), 265-268. [Russian] Translated in: Soviet Mathematics - Doklady 1 (1960), 829832.

d) (With Ya.I. Hurgin and B.S. Tsybakov). An approximate computation of the capacity of radio channels with random parameters, In: Trudy Vsesoyuznogo Soveschachnia po Teorii Veroyatnosteii $i$ Matematicheskoi Statistike, Izdatel'stvo AN Arm SSR (Yerevan, 1958)., (1960), 164-171. [Russian]

e) (With A.M. Jaglom and I.M. Jaglom). Theory of information and linguistics, Voprosy Yazykoznaniya 9:1 (1960), 100-110. [Russian]

\section{1}

a) Mathematical method in linguistics, Matematicheskoe Prosveschenie 6 (1961), 37-60. [Russian]

b) Mathematical problems in Shannon theory of optimal coding of information, In: Problemy Peredachi Informatisii 10, Izdatel'stvo AN SSSR, Moscow (1961), 63-107. [Russian] Translated in: Proc. Fourth Berkeley Symp. on Math. Stat. and Prob. 1960 (1962), 211-252.

\section{2}

a) Optimal binary codes for small rates of transmission of information, Teoriya Veroyat. $i$ ee Primeneniya 7:2 (1962), 208-213. [Russian] Translated in: Theory Prob. Its Appl. 7 (1962), 199204.

b) Asymptotic estimates of the probability of error for transmission of messages over a discrete memoryless communication channel with a symmetric transition probability matrix, Teoriya Veroyat. $i$ ee Primeneniya 7:3 (1962), 283-311. [Russian] Translated in: Theory Prob. Its Appl. 7 (1962), 270-300.

c) Asymptotic estimates of the probability of error for the transmission of messages over a memoryless channel with feedback, Problemy Kibernetiki 3 (1962), 161-168. [Russian]

d) (With B.S. Tsybakov). Information transmission with additional noise, IEEE Trans. Inform. Theory 8 (1962), 293-304.

\section{3}

a) Asymptotic optimality of group and systematic codes for some channels, Teoriya Veroyat. $i$ ee Primeneniya 8:1 (1963), 52-66. [Russian] Translated in: Theory Prob. Its Appl. 8 (1963), 4760.

b) Individual methods for the transmission of information for discrete channels without memory and messages with independent components, Doklady AN SSSR 148:6 (1963), 1245-1248. [Russian] Translated in: Soviet Mathematics - Doklady 4 (1963), 253-256.

c) Unified methods for the transmission of information: a general case, Doklady AN SSSR 149:1 (1963), 16-19. [Russian] Translated in: Soviet Mathematics - Doklady 4 (1963), 257-259.

d) (With M.S. Pinsker and A.N. Shiryaev). An application of the entropy to problems of signal detecting against a background of noise, Litovskii Matematicheskii Sbornik 3:1 (1963), 107-122. [Russian]

e) (With R.A. Minlos). Possibilities of applications of limit theorems of probability theory to some physical problems, In: Predel'nye Teoremy Teorii Veroyatnosteii Izdatel'stvo FAN, Tashkent (1963), 15-37. [Russian] 


\section{4}

a) On the Wozencraft-Reiffen method of sequential decoding, Problemy Kibernetiki 12 (1964), 113-123. [Russian]

b) Investigation of conditions for the asymptotic existence of the configurational integral of the Gibbs distribution, Teoriya Veroyat. $i$ ee Primeneniya 9:4 (1964), 626-643. [Russian] Translated in: Theory Prob. Its Appl. 9 (1964), 566-581.

c) Methods of probability theory in statistical physics, In: Trudy Zimnei Shkoly po Teorii Veroyatnosteii i Matematicheskooii Statistike, Izdatel'stvo KGU, Kiev (Uzhgorod, 1964), 221263. [Russian]

\section{5}

a) Existence of a phase transition in the two-dimensional Ising models, Doklady AN SSSR 165:5 (1965), 1046-1048. [Russian] Translated in: Soviet Physics - Doklady 10 (1965), 111-113.

b) Existence of a phase transition in two and three dimensional Ising models, Teoriya Veroyat. $i$ ee Primeneniya 10:2 (1965), 209-230. [Russian] Translated in: Theory Prob. Its Appl. 10 (1965), 193-213.

\section{6}

a) Existence of phase transitions in models of a lattice gas, Proc. Fifth Berkeley Symp. on Math. Stat. and Prob. 19653 (1966), 73-87.

b) Theory of optimal coding of information, In: Kibernetiku - na Sluzhbu Kommunizmu 3 Energija, Moscow (1966), 13-45. [Russian]

\section{7}

a) (With R.A. Minlos). Existence and continuity of the presure in classical statistical physics, Teoriya Veroyat. i ee Primeneniya 12:4 (1967), 595-618. [Russian] Translated in: Theory Prob. Its Appl. 12 (1967), 535-559.

b) Shannon theorems for channels with synchronization errors, Problemy Peredachi Informatisii 3:4 (1967), 18-36. [Russian] Translated in: Problems of Inform. Trans. 3:4 (1967), 11-26.

\section{8}

a) The description of a random field by means of conditional probabilities and conditions of its regularity, Teoriya Veroyat. i ee Primeneniya 13:2 (1968), 201-229. [Russian] Translated in: Theory Prob. Its Appl. 13 (1968), 197-213.

b) Gibbsian random fields for lattice systems with pairwise interactions, Funktsional'nyi Analis $i$ ego Prilozheniya 2:2 (1968), 31-43. [Russian] Translated in: Functional Analysis and Its Appl. 2 (1968), 292-301.

c) The problem of uniqueness of a Gibbsian random field and the problem of phase transition, Funktsional'nyi Analis $i$ ego Prilozheniya 2:2 (1968), 44-57. Translated in: Functional Analysis and Its Appl. 2 (1968), 302-312.

d) (With N. Vvedenskaja). Calculation on a computer of the capacity of communication channels with exclusion of symbols, Problemy Peredachi Informatisii 4:3 (1968), 92-95. [Russian] Translated in: Probl. of Infor. Trans. 4:3 (1968), 92-95.

\section{9}

a) Gibbsian fields. General case, Funktsional'nyi Analis i ego Prilozheniya 3:1 (1969), 27-35. [Russian] Translated in: Functional Analysis and Its Appl. 3 (1969), 22-28.

b) (With I.I. Pjatetskii-Shapiro and N.B. Vasiljev). Markov processes on an infinite product of discrete spaces, In: Proc. of the Soviet-Japanese Symposium in Prob. Theory (Khabarovsk, June 1968), Khabarovski-Novosibirsk (1969), 3-30. [Russian]

c) (With M.S. Pinsker). Memory increases transmission capacity. Problemy Peredachi Informatsii 5:1 (1969), 94-95. [Russian] Translated in: Problems of Inform. Trans. 5 (1969), 77-78.

1970

a) Gibbsian random fields for particles without hard core, Teoreticheskaya i Matematicheskaya 
Fizika 4:1 (1970), 101-118. [Russian]

b) Prescribing a system of random variables with conditional distributions, Teoriya Veroyat. $i$ ee Primeneniya 15:3 (1970), 469-497. [Russian] Translated in: Theory Prob. Its Appl. 15 (1970), 458-486.

c) Unified methods of optimal quanitization of messages, Problemy Kibernetiki 22 (1970), 107156. [Russian].

1971

a) Markov processes with a large number of locally interacting components: Existence of a limit processes and their ergodicity, Problemy Peredachi Informatsii 7:2 (1971), 70-87. [Russian] Translated in: Problems of Inform. Trans. 7 (1971), 149-164.

b) Markov processes with many locally interacting components - the reversible case and some generalizations, Problemy Peredachi Informatsii 7:3 (1971), 57-66. [Russian] Translated in: Prob. of Inform. Trans. 7 (1971), 235-241.

c) (With R.A. Minlos and Yu.M. Suhov). A review of some recent results, In: Statisticheskaya Mehanika. Strogie Resul'taty Mir, Moscow (1971), 314-361. [Russian] (A complement to the Russian translation of the book: D. Ruelle, Statistical Mechanics. Rigorous Results, Benjamin, New York 1969.)

1972

a) Asymptotic behavior of Gibbsian distributions for lattice systems and its dependence on a form of a volume, Teoreticheskaya $i$ Matematicheskaya Fizika 12 (1972), 669-711. [Russian] Translated in: Theoretical and Math. Physics 12 (1972), 669-711.

b) Gibbs states describing a coexistence of phases for the three-dimensional Ising model, Teoriya Veroyatnostei $i$ ee Primeneniya 17:4 (1972), 619-639. [Russian] Translated in: Theory of Prob. and Its Appl. 17 (1972), 582-600; reprinted in: Math. Prob. of Statistical Mechanics, World Scientific, Singapore 1991.

c) (With S.I. Gelfand). Complexity of asymptotically optimal code realization by constant depth schemes, Prob. of Control and Inform. Theory 1 (1972), 197-215.

d) Survey of Soviet research in information theory, IEEE Trans. Inform. Theory 18 (1972), 703724 .

\section{3}

a) An investigation of Gibbsian states for three-dimensional lattice systems, Teoriya Veroyat. $i$ ee Primeneniya 18:2 (1973), 261-279. [Russian] Translated in: Theory Prob. Its Appl. 18 (1973), 253-271. Reprinted in: Math. Problems of Statistical Mechanics, World Scientific, Singapore 1991.

b) Analiticity of correlation functions in one-dimensional classical systems with slowly decreasing potentials, Commum. Math. Phys. 32 (1973), 269-289.

c) (With R.A. Minlos). Construction of one-dimensional quantum field via a continuous Markov field, Funktsional'nyi Analis $i$ ego Prilozheniya 7:4 (1973), 81-82. [Russian] Translated in: Functional Analysis and Its Appl. 7 (1973), 324-325.

d) Mathematization of linguistics, Izvestia Academii Nauk SSSR, Ser. Literatura, Linguistika 32:5 (1973), 438-441. [Russian]

e) (With S.I. Gelfand and M.I. Pinsker). On complexity of coding, In: Proc. Second International Symposium on Information Theory (Tsahkadzor, Armenia, September 1971) Academia Kiado, Budapest (1973), 177-184.

1974

a) Conditions of absence of phase transitions in one-dimensional classical systems, Matematicheskii Sbornik 93:1 (1974), 29-49. [Russian] Translated in: Math. of the USSR - Sbornik 22 (1974), 28-48.

b) Analyticity of correlation functions in one-dimensional classical systems with a slowly power decrease of potential, Mathematicheskii Sbornik 94:1 (1974), 16-48. [Russian] Translated in: 
Math. of the USSR - Sbornik 23 (1974), 13-44.

c) (With V.M. Gertski). Gibbsian states in a lattice model with the two-step interaction, Funktsional'nyi Analis $i$ ego Prilozheniya 8:3 (1974), 12-25. [Russian] Translated in: Functional Analysis and Its Appl. 8 (1974), 201-211.

d) (With B.S. Nakhapetjan). Strong convexity of the pressure for lattice systems of classical statistical physics, Teoreticheskaya i Matematicheskaya Fizika 20:2 (1974), 223-234. [Russian] Translated in: Theoretical and Math. Physics 20 (1974), 782-790.

\section{5}

a) (With S.B. Shlosman). Absence of breakdown of continuous symmetry in two-dimensional models of statistical physics, Commun. Math. Phys. 42 (1975), 31-40.

b) (With S.Z. Stambler). Coding theorems for classes of arbitrary varying discrete memoryless channels, Problemy Peredachi Informatisii 11:2 (1975), 3-22. [Russian] Translated in: Problems of Inform. Trans. 11 (1975), 97-112.

c) (With R.A. Minlos). Quotient measures on measurable spaces, Trudy Moskovskogo Matematicheskogo Obschchestva 32 (1975), 77-92. [Russian] Translated in: Trans. of the Moscow Math. Society 32 (1975), 73-88.

\section{6}

a) (With R.M. Minlos). An investigation of properties of generalized Gaussian random fields, In: Zadachi Mekhaniki i Matematicheskoii Fiziki Nauka, Moscow (1976), 117-165. [Russian] Translated in: Selecta Mathematica Sovietica 1 (1981), 215-263.

b) (With Yu.M. Sukhov). Asymptotical investigation of star-shape message switching networks with a large number of radial rays, Problemy Peredachi Informatsii 12:1 (1976), 70-94. [Russian] Translated in: Problems of Inform. Trans. 12 (1976), 49-65.

c) (With S.A. Pirogov). Theory of random fields, Proc. of the 1975 IEEE-USSR Joint Workshop on Inform. Theory, IEEE Press, New York (1976), 39-49.

\section{7}

a) (With B. Tirozzi). The central limit theorem and the problem of equivalence of ensembles, Commun. Math. Phys. 54 (1977), 173-192.

b) (With J. Fritz). Nonequilibiruym dynamics of one-dimensional infinite particle systems with a singular interaction, Commun. Math. Phys. 55 (1977), 275-292.

c) (With J. Fritz). Nonequilibrium dynamics of two-dimensional infinite particle systems with a singular interaction, Commun. Math. Phys. 57 (1977), 67-81.

d) (With R.A. Minlos). Polynoms in linear random functions, Uspekhi Matematicheskih Nauk 32:2 (1977), 67-122. [Russian] Translated in: Russian Math. Surveys - Uspekhi 32:2 (1977), 71-127.

e) (With S.I. Ortjukov). Lower bound for the redundancy of self-correcting arrangements of unreliable functional elements, Problemy Peredachi Informatsii 13:1 (1977), 82-89. [Russian] Translated in: Problems of Inform. Trans. 13 (1977), 59-65.

f) (With S.I. Ortjukov). Upper bound for the redundancy of self-correcting arrangements of unreliable functional elements, Problemy Peredachi Informatsii 13:3 (1977), 56-76. [Russian] Translated in: Problems of Inform. Trans. 13 (1977), 203-218.

\section{8}

a) Automodel generalized random fields and their renorm-groups, In: Mnogokomponentye Sluchaiinye Sistemy. Nauka, Moscow (1978), 179-213. [Russian] Translated in: Multicomponent Random Systems (ed. by R.L. Dobrushin and Ya.G. Sinai). Advances in Probability and Related Topics 6 (1980), Marcel Dekker, New York, 153-198.

b) (With S.B. Shlosman). Nonexistence of one and two-dimensional Gibbs fields with a noncompact group of continuous symmetries, In: Mnogokomponentnye Sluchaiinye Sistemy. Nauka, Moscow (1978), 214-223. [Russian] Translated in: Multi-component Random Systems (ed. by r.L. Dobrushin and Ya.G. Sinai). Advances in Probability and Related Topics 6 (1980), 
Marcel Dekker, New York, 199-210.

c) (With Yu.M. Sukhov). On the problem of the mathematical foundation of the Gibbs postulate in classical statistical mechanics. In: Mathematical Problems in Theoretical Physics (ed. by G. Dell'Antonio, S. Doplicher, and G. Iona-Lasinio). Lecture Notes in Physics 80, SpringerVerlag (1978), Berlin, 325-340.

d) (With R.A. Minlos). Polynomials of a generalized random field and its moments, Teoriya Veroyat. $i$ ee Primeneniya 23:4 (1978), 715-730. [Russian] Translated in: Theory Prob. Its Appl. 23 (1978), 686-699.

\section{9}

a) Gaussian and their subordinated self-similar random generalized fields, Ann. Prob. 7 (1979), 128.

b) (With D. Surgailis). On the innovation problem for Gaussian Markov random fields, $Z$. Wahrsch. Verw. Geb. 49 (1979), 275-291.

c) The Vlasov equation, Funktsional'nyi Analisi ego Prilozheniya 13:2 (1979), 48-58. [Russian] Translated in: Functional Analysis and Its Appl. 13 (1979), 115-123.

d) (With Yu.M. Sukhov). Time asymptotics for some degenerate models of time evolution for system with infinitely many particles, Itogi Nauki i Tehniki. Ser. Sovremennye Problemy Matamatiki 14 VINITI, Moscow (1979), 147-254. [Russian] Translated in: J. Soviet Math. 16 (1981), 1277-1340.

e) (With P. Major). Non-central limit theorems for nonlinear functions of Gaussian fields, $Z$. Wahrsch. Verw. Geb. 50 (1979), 27-52.

f) (With V.V. Prelov). Asymptotic approach to the investigation of message switching networks of a linear structure with a large number of centers, Problemy Peredachi Informatisii 15:1 (1979), 61-73. [Russian] Translated in: Problems of Information Trans. 15 (1979), 46-55.

g) (With M.Ya. Kelbert). Local additive functionals of Gaussian random fields, Uspekhi Matematicheskih Nauk 34:5 (1979), 223-224. [Russian] Translated in: Russian Math. Surveys - Uspekhi 34:5 (1979), 237-238.

\section{0}

a) Gaussian random fields: Gibbsian point of view, In: Multi-component Random Systems (ed. by R.L. Dobrushin and Ya.G. Sinai), Advances in Probability and Related Topics 6 (1980), Marcel Dekker, New York, 119-152. [Russian]

b) (With Ya.G. Sinai). Mathematical problems in statistical mechanics, Soviet Scientific Reviews, Section C: Mathematical Physics Reviews 1 (1980), 55-106.

c) (With C. Boldrighini and Yu.M. Suhov). Hydrodynamical limit for a degenerate model of classical statistical mechanics, Uspekhi Matematicheskih Nauk 35:4 (1980), 152. [Russian]

d) (With C. Boldrighini and Yu.M. Suhov). Hydrodynamics of one-dimensional hard rods, Uspekhi Matematicheskih Nauk 35:5 (1980), 252-253. [Russian]

e) (With C. Boldrighini and Yu.M. Suhov). Time Asymptotic for Some Degenerate Models of Time Evolution for System with Infinitely Many Particles, University Publishing House, Camerino 1980.

\section{1}

a) (With E.A. Pechersky). Uniqueness conditions for finitely dependent random fields, In: Random Fields (ed. by J. Fritz, J.L. Lebowitz and D. Szasz) 1, North Holland, Amsterdam (1981), 223-262.

b) (With S.B. Shlosman). Phases corresponding to minima of the local energy, Selecta Mathematica Sovietica 1 (1981), 317-338.

c) (With P. Major). On asymptotical behavior of some selfsimilar random fields, Selecta Mathematica Sovietica 1 (1981), 265-291.

d) (With R.M. Gray and D.S. Ornstein). Block synchronization, sliding-block coding, invulnerable sources and zero error codes for discrete noisy channels, Ann. of Prob. 8 (1981), 639-674. 


\section{2}

a) (With R. Siegmund-Schultze). The hydrodynamic limit for systems of particles with independent evolution, Math. Nachr. 105 (1982), 199-224.

b) Hydrodynamic limit approach: Some caricatures, In: Vzaimodeiistvuyuschchie Markovskie Protsessy $i$ ih Primeneniya $k$ Matematicheskomu Modelirovaniyu Biologicheskih Sistem, Puschchino (Moscow Region) (1982), 7-20. [Russian]

\section{3}

a) (With E.A. Pechersky). A criterion of the uniqueness of Gibbsian fields in the noncompact case, In: Prob. Theory and Math. Statistics: Proc. of the Fourth USSR-Japan Symposium (ed. by K. Ito and J.V. Prokhorov), Lecture Notes in Math 1021, Springer-Verlag, Berlin (1983), $97-110$.

b) (With C. Boldrighini and Yu.M. Suhov). One dimensional hard rod caricature of hydrodynamics, J. Stat. Phys. 31 (1983), 577-615.

c) (With M.Ya. Kelbert). Local additive functionals on Gaussian random fields, Teoriya Veroyat. i ee Primeneniya 28:1 (1983), 32-44. [Russian] Translated in: Theory Prob. Its Appl. 28 (1984), 35-47.

d) (With M.Ya. Kelbert). Stationary local additive functionals of Gaussian fields, Teoriya Veroyat. $i$ ee Primeneniya 28:3 (1983), 489-503. Translated in: Theory Prob. Its Appl. 28 (1984), 515-529.

\section{5}

a) (With S.B. Shlosman). Constructive criterion for the uniqueness of Gibbs field, In: Statistical Physics and Dynamical Systems. Rigorous Results, (ed. by J. Fritz, A. Jaffe and D. Szasz), Progress in Physics 10, Birkhauser, Boston (1985), 347-370.

b) (With S.B. Shlosman). Completely analytical Gibbs fields, In: Statistical Physics and Dynamical Systems. Rigorous Results (ed. by J. Fritz, A. Jaffe and D. Szasz), Progress in Physics 10, Birkhauser, Boston (1985), 371-404.

c) (With I. Kolafa and S.B. Shlosman). Phase diagram of the two-dimensional Ising antiferromagnet, Commun. Math. Phys. 102 (1985), 81-103.

d) (With M.G. Avetisjan). A condition of the linear regularity for vector random fields, Problemy Peredachi Informatsii 21:4 (1985), 76-82. [Russian] Translated in: Problems of Inform. Trans. 21:4 (1985), 308-313.

e) (With S.B. Shlosman). The problem of translation invariance of Gibbs states at low temperature, Soviet Scientific Reviews, Section C: Mathematical Physics Reviews 5 (1985), 53-195.

f) (With Ya.G. Sinai and Yu.M. Sukhov). Dynamical systems of statistical mechanics, In: Itogi Nauki $i$ Tehniki. Ser. Sovemennye Problemy Matematiki. Fundamentalnye Napravleniya 2 Dinamicheskie Systemy 2, VINITI, Moscow (1985), 235-284. [Russian] Translated in: Dynamical Systems II, Encyclopedia of Mathematical Sciences, 2 (ed. by Ya.G. Sinai), Springer-Verlag, Berlin (1989), 208-254.

\section{6}

a) (With M. Zahradnik). Phase diagrams for continuous-spin models. An extension of the Pirogov-Sinai theory, In; Mathematical Problems of Statistical Mechanics and Dynamics, D. Reidel, Dordrecht (1986), 1-124.

b) (With L.A. Bassalygo), Uniqueness of a Gibbs field with random potential-an elementary approach, Teoriya Veroyatnostei $i$ ee Primeneniya 31:4 (1986), 651-670. [Russian] Translated in: Theory of Prob. and Its Appl. 31 (1987), 572-589.

c) (With A. Pellegrinotti, Yu.M. Suhov and L. Triolo). One-dimensional harmonic lattice caricature of hydrodynamics, J. Stat. Phys. 43 (1986), 571-608.

1987

a) (With S.B. Shlosman). Completely analytical interactions: constructive description, J. Stat. Phys. 46 (1987), 983-1014. 
b) Induction on volume and no cluster expansion, In: Proc. VIII International Congress on Mathematical Physics (ed. by M. Mebkhout and B. Seneor), World Scientific, Singapore (1987), 73-91.

c) (With L.A. Bassalygo), $\epsilon$-entropy of Gibbsian fields, Problemy Peredachi Informatssi 23:1 (1987), 3-15. [Russian] Translated in: Problems of Inform. Trans. 23 (1987), 1-10.

d) Theory of information, In: A.N. Kolmogorov Izbrannyie Trudy. Teoriya Informatsii $i$ Teoriya Algoritmov, Moscow, Nauka (1987), 254-257. [Russian]

e) Switching networks, Gibbsian fields - interconnections, In: Proc. 1st World Congress of the Bernoulli Society, Tashkent (1986), VN Sci. Press, Utrecht (1987), 377-393.

\section{8}

a) A new approach to the analysis of Gibbs perturbations of Gaussian fields, Selecta Mathematica Sovieta 7 (1988), 221-277.

b) (With M.R. Matirosjan). Non-finite perturbation of Gibbsian fields, Teoreticheskaya $i$ Matematicheskaya Fizika 74:1 (1988), 10-20. [Russian] Translated in: Theoretical and Mathematical Physics 74 (1988), 10-20.

c) (With M.R. Martirosjan). A possibility of high-temperature phase transition due to many particle character of potential, Teoreticheskaya i Matematicheskaya Fizika 75:2 (1988), 163169. [Russian] Translated in: Theoretical and Mathematical Physics 75 (1988), 443-448.

d) (With J. Fritz and Yu.M. Suhov). A.N. Kolmogorov, the founder of the theory of reversible Markov processes, Uspekhi Matematicheskih Nauk 43:6 (1988), 167-188. [Russian] Translated in: Russian Mathematical Surveys - Uspekhi 43:6 (1988), 157-182.

e) (With A. Pellegrinotti, Yu.M. Suhov and L. Triolo). One-dimensional harmonic lattice caricature of hydrodynamics: second approximation, J. Stat. Phys. 52 (1988), 423-439.

\section{9}

a) Caricatures of hydrodynamics, In: Proc. IX International Congress on Math. Physics (ed. by B. Simon, A. Truman and I.M. Davies), Adam Higler, Bristol (1989), 117-132.

b) (With R. Kotecky and S.B. Shlosman). Equilibrium crystal shapes - a microscopic proof of the Wulff construction. In: Proc. of XXIV Karpacz Winter School. Stochastic Methods in Math. Physics, World Scientific, Singapore (1989), 221-229.

\section{0}

a) (With M.Ya. Kelbert, A.N. Rybko and Yu.M. Suhov). Qualitative methods of queueing network theory, In: Stochastic Cellular Systems: Ergodicity, Memory, Morphogenesis (ed. by R.L. Dobrushin, V.M. Kryukov and A.L. Toom), University Press, Manchester (1990), 183224.

b) (With A. Pellegrinotti and Yu.M. Suhov). One-dimensional harmonic lattice caricature of hydrodynamics. The higher corrections, J. Stat. Phys. 61 (1990), 387-402.

c) (With V. Warstat). Completely analytic interactions with infinite values, Prob. Theory Rel. Fields 84 (1990), 335-359.

\section{1}

a) (With F. Sokolovskii). Higher order hydrodynamic equations for a system of independent random walks, In: Random Walks, Brownian Motion and Interacting Particle Systems. A Festschrift in Honor of Frank Spitzer (ed. by R. Durrett and H. Kesten), Birkhauser, Boston (1991), 231-254.

\section{2}

a) (With R. Kotecky and S.B. Shlosman). Wuff Construction: A Global Shape from Local Interaction, Translations of Mathematical Monographs 104, AMS, Providence RI 1992.

b) (With S.B. Shlosman). Thermodynamics inequalities for the surface tension and the geometry of the Wulff construction, In: Ideas and Methods of Math. Analysis, Stoch. and Appl. In Memory of R. Hoegh-Krohn (ed. by S. Albeverio, J.E. Fenstad, H. Holden and J.E. Lindstron) 
21, Cambridge University Press, Cambridge (1992), 461-483.

c) (With S.B. Shlosman). Large deviations behavior of statistical mechanics models in multiphase regime, In: Proc. X International Congress on Math. Physics (ed. by K. Schmüdzen), Springer-Verlag, Berlin (1992), 328-333.

\section{3}

a) A formula of full semi-invariants, In: Cellular Automata and Cooperative Systems (ed. by N. Boccara, E. Goles, S. Martinez, and P. Picco), Kluwer, Dordrecht (1993), 135-140.

b) On the way to the mathematical foundations of statistical mechanics, In: Statistical Mechanics and Fractals, Lecture Notes in Mathematics 1567, Springer-Verlag (1993), 1-37.

c) A statistical behavior of shapes of boundaries of phases, In: Phase Transitions: Mathematics, Physics, Biology, etc. (ed. by K. Kotecky), World Scientific, Singapore (1993), 60-70.

d) (With R. Kotecky and S.B. Shlosman). A microscopic justification of the Wulff construction, J. Stat. Phys. 72 (1993), 1-14.

\section{4}

a) Estimates of semi-invariants for the Ising model at low temperatures, Preprint 125, ESI, Vienna.

b) (With E.A. Pechersky). Large deviations for tandem queueing systems, J. Appl. Math. Stoch. Anal. 7 (1994), 301-330.

c) (With S.B. Shlosman). Large and moderate deviations in the Ising model, In: Probability Contributions to Statistical Mechanics (ed. by R.L. Dobrushin), Advances in Soviet Mathematics 20, AMS, Providence, RI (1994), 91-219.

d) (With S.B. Shlosman). Droplet condensation in the Ising model: moderate deviations point of view, In: Probability and Phase Transition (ed. by G. Grimmett), Kluwer, Dordrecht (1994), $17-35$.

e) (With V.M. Blinovsky). Process level large deviations for a class of piecewise homogeneous random walks, In: The Dynkin Festschrift. Markov Processes and Their Applications (ed. by M.I. Freidlin), Progress in Probability 50, Birkhauser, Boston (1994), 1-60.

\section{5}

a) (With O.O. Hryniv). On fluctuations of Wulff's shape in the two dimension Ising model, Uspekhi Matematicheskih Nauk 50:6 (1995), 177-178. [Russian] Translated in: Russian Math. Surveys-Uspekhi 50:6 (1995), 1262-1264.

b) (With O.O. Hryniv). On fluctuations of the phase boundary in the 2D Ising ferromagnet, Preprint 355, ESI, Vienna.

\section{6}

a) (With N.D. Vvedenskaya and F.I. Karpelevich). A queueing system with selection of the shortest of two queues: An asymptotical approach, Problems of Info. Trans. 32 (1996), 15-27.

b) (With O.O. Hryniv). Fluctuations of shapes of large areas under paths of random walks, Prob. Theory Rel. Fields 105 (1996), 423-458.

\section{Acknowledgements}

The authors would like to thank L. Bassalygo, C. Boldrighini, G. Dobrushina, B. Gurevich, A. Jaffe, R. Kotecky, L. Philippova, M. Pinsker, Ya. Sinai, S. Shlosman, A. Tempelman, B. Tsybakov, and N. Vvedenskaya for valuable comments on various matters discussed in the article. The hospitality of the Erwin-Schrödinger Institute and of the organizers of the Dobrushin Memorial Conference, "Statistical Mechanics as a Branch of Probability Theory" (Vienna, September 16-20, 1996) is particularly appreciated. Special thanks are due to Sarah SheaSimonds for correcting the style of the paper. 


\section{References}

[1] Aizenman, M., Translation invariance and instability of phase coexistence in two-dimensional Ising system, Commun. Math. Phys. 73 (1980), 83-94.

[2] Aizenman, M., Goldstein, S. and Lebowitz, J.L., Ergodic properties of an infinite one-dimensional hard rod system, Commun. Math. Phys. 39 (1975), 289-301.

[3] Aizenman, M., Jaffe, A., Khanin, K., Lebowitz, J.L., Lieb, E., Mazel, A., Shlosman, S., Sinai, Ya. and Stroock, D., Roland Dobrushin (1929-1995), IAMP News Bulletin, January (1996), 2.

[4] Anantharam, V., Uniqueness of stationary ergodic fixed point for a $\cdot / M / k$ node, Ann. Appl. Prob. 3 (1993), 154-173.

[5] Averintsev, M.B., A way of describing random fields with a discrete argument, Problems of Inform. Trans. 6 (1970), 169-175.

[6] Averintsev, M.B., Description of Markovian random fields by Gibbsian conditional probabilities, Theory of Probability and its Applications 17 (1972), 20-23.

[7] Averintsev, M.B., On some classes of Gibbsian random fields, In: Locally Interacting Systems and Their Applications in Biology (ed. by R.L. Dobrushin, V.I. Kryukov and A.L. Toom), Lecture Notes in Mathematics 653, Springer-Verlag, Berlin (1978), 91-98.

[8] Basis, V.Ya., Infinite-dimensional Markov processes with almost local interaction of components, Theory of Prob. and its Appl. 21 (1976), 706-720.

[9] Bassalygo, L.A., Malyshev, V.A., Minlos, R.A., Ovseevich, I.A., Pechersky, E.A., Pinsker, M.S., Prelov, V.V., Rybko, A.N., Suhov, Y.M., and Shlosman, S.B., Roland L'vovich Dobrushin, Problemy Peredachi Informatsii 32:3 (1996), 3-24. [Russian]

[10] Berezin, F.A. and Sinai, Ya.G., Existence of a phase transition for the lattice gas with interparticle attraction, Trans. of the Moscow Math. Society 17 (1967), 219-236.

[11] Berezner, S.A. and Malyshev, V.A., The stability of infinite-server networks with random routing, J. Appl. Prob. 26 (1989), 363-371.

[12] Bernstein, S.N., Sur la théorème du calcul de probabilités, Math. Ann. 85 (1922), 237-241.

[13] Bernstein, S.N., Sur l'éxtension du théorème limite du calcul des probabilités aux sommes des quantités dépendantes, Math. Ann. 97 (1926), 1-59.

[14] Bernstein, S.N., Sur les sommes des quantités dépendantes, Izvestiia AN SSSR, VI seriya 15-17 (1928), 1459-1478.

[15] Bernstein, S.N., Détérmination d'une limite intérieure de la dispersion des sommes de grandeurs liées en chaine singulère, Matematicheskii sbornik 1:1 (1936), 29-38.

[16] Bogoliubov, N.N., The equations of hydrodynamics in statistical mechanics, In: N.N. Bogoliubov, Izbrannye Trudy 2, Naukova Dumka, Kiev (1970), 258-276. [Russian]

[17] Bramson, M., Instability of FIFO queueing networks, Ann. Appl. Prob. 4 (1994), 414-431.

[18] Bramson, M., Instability of FIFO queueing networks with quick service times, Ann. Appl. Prob. 4 (1994), 693-718.

[19] Fröhlich, J. and Simon, B., Pure states for general $P(\phi)_{2}$ theories: construction, regularity and variational equality, Ann. Math. 105 (1977), 493-526.

[20] Gallavotti, G. and Miracle-Sole, S., Statistical mechanics of lattice systems, Commun. Math. Phys. 5 (1967), 317-324.

[21] Gallavotti, G., The phase separation line in the two-dimensional Ising model, Commun. Math. Phys. 27 (1972), 103-136.

[22] Gerogii, H.-O., Gibbs Measures and Phase Transitions, De Gruyter, Berlin 1988.

[23] Gertsik, V.M., Conditions for the non-uniqueness of the Gibbs state for lattice models having finite interaction potential, Mathematics of the USSR - Izvestija 10 (1976), 429-443.

[24] Ginibre, J., Grossmann, A. and Ruelle, D., Condensation of lattice gas, Commun. Math. Phys. 3 (1966), 187-193.

[25] Glimm, J. and Jaffe, A., Quantum Physics. A Functoinal Point of View, Springer-Verlag, New York 1987. 
[26] Glimm, J., Jaffe, A. and Spencer, T., Phase transitions for $\phi_{2}^{4}$ quantum fields, Commun. Math. Phys. 45 (1975), 203-216.

[27] Glimm, J., Jaffe, A. and Spencer, T., A convergent expansion about mean-field theory I, Ann. Physics 101 (1976), 610-630.

[28] Glimm, J., Jaffe, A. and Spencer, T., A convergent expansion about mean-field theory II, Ann. Physics 101 (1976), 631-669.

[29] Glimm, J., Jaffe, A. and Spencer, T., Existence of phase transitions for quantum fields, In: Les Méthodes Mathematique de la Théorie Quantique des Champes, Marseille 1976 (ed. by F. Guerra, D. Robinson and R. Stora), Editions du Centre National de la Recherche Scientifique, Paris 1976.

[30] Griffiths, R.B., Peierls' proof of spontaneous magnetization of a two-dimesnional Ising models, Phys. Rev. A136 (1964), 437-439.

[31] Grimmett, G.R., A theorem about random fields, Bull. London Math. Soc. 5 (1973), 81-84.

[32] Gross, D. and Harris, C.M., Fundamentals of Queueing Theory, Wiley, Chichester 1985.

[33] Gurevich, B.M., Asymptotically additive integrals of the motion of particles with pairwise interaction, Trans. of the Moscow Math. Society 52 (1990), 177-232.

[34] Gurevich, B.M., Asymptotically additive integrals of motion for particles with non-pairwise interaction in dimension one, In: Probability Contributions to Statistical Mechanics (ed. by R.L. Dobrushin), Advances in Soviet Math. 20, AMS, Providence, RI (1994), 221276 .

[35] Gurevich, B.M., Ibragimov, I.A., Minlos, R.A., Ovseevich, I.A., Oseledets, A.I., Pinsker, M.S., Prelov, V.V., Prohorov, Yu.V., Sinai, Ya.G., Holevo, A.S., and Shiryaev, A.L., Roland L'vovich Dobrushin (VII 20, 1929-XI 12, 1995), Teoriya Veroyatnosteii $i$ ee Primeneniia 41:1 (1996), 164-169. [Russian].

[36] Gurevich, B.M. and Suhov, Yu.M., Stationary solutoins of the Bogoliubov hierarchy equations 1, Commun. Math. Phys. 49 (1976), 69-96.

[37] Gurevich, B.M. and Suhov, Yu.M., Stationary solutions of the Bogoliubov hierarchy equaltions 2, Commun. Math. Phys. 54 (1977), 81-96.

[38] Gurevich, B.M. and Suhov, Yu.M., Stationary solutoins of the Bogoliubov hierarchy equations 3, Commun. Math. Phys. 56 (1977), 225-236.

[39] Gurevich, B.M. and Suhov, Yu.M., Stationary solutions of the Bogoliubov hierarchy equations 4, commun. Math. Phys. 84 (1982), 333-376.

[40] Higuchi, Y., On the absence of non-translation invariant Gibbs states for the two-dimensional Ising model, In: Random Fields (ed. by J. Fritz, J.L. Lebowitz and D. Szasz), Proc. of the Esztergom Conference (1979) 1, North-Holland, Amsterdam, 1981.

[41] Jackson, J.R., Networks of waiting times, Operational Research 5 (1957), 518-527.

[42] Jackson, J.R., Jobshop-like queueing systems, Management Sci. 10 (1965), 131-142.

[43] Karpelevich, F.I., Kelbert, M.Ya., and Suhov, Yu.M., Higher-order Lindley equations, Stoch. Proc. Appl. 53 (1994), 65-96.

[44] Kaufman, B., Crystal statistics 2. Partition function evaluated by spinor analysis, Phys. Rev. 76 (1949), 1232-1243.

[45] Kelbert, M.Ya., Kontsevich, M.A. and Rybko, A.N., On Jackson networks on denumerable graphs, Theory of Prob. and Its Appl. 33 (1989), 358-361.

[46] Kelbert, M.Ya. and Suhov, Yu.M., Existence and uniqueness conditions for a random field describing the state of a switching network, Problems of Inform. Trans. 19 (1983), 289304.

[47] Kelbert, M.Ya. and Suhov, Yu.M., Weak dependence of the random field describing state of a switching network with small transit flows, Problems of Inform. Trans. 21 (1985), 237-245.

[48] Kelbert, M.Ya. and Suhov, Yu.M., Mathematical problems in teh theory of queueing networks, J. Soviet Math 50 (1990), 1527-1600.

[49] Kelly, F.P., Networks of queues, Adv. Appl. Prob. 8:2 (1976), 416-432. 
[50] Kelly, F.P., Reversibility and Stochastic Networks, Wiley, New York 1979.

[51] Kozlov, O.K., Gibbsian description of point random fields, Theory Prob. Its Appl. 21 (1976), 339-531.

[52] Kozlov, O.K., Consistent systems of conditional distributions of a random field, Probl. of Inform. Trans. 13 (1977), 218-227.

[53] Lanford, O.E., The classical evolution of one-dimensional systems of infinitely many particles I. An existence theorem, commun. Math. Phys. 9 (1968), 176-191.

[54] Lanford, O.E., The classical evolution of one-dimensional systems of infinitely many particles II. Kinetic theory, Commun. Math. Phys. 11 (1969), 257-292.

[55] Lanford, O.E., Lebowitz, J.L. and Lieb, E.H., Time evolution of infinite anharmonic systems, J. Stat. Phys. 16 (1977), 453-461.

[56] Lanford, O.E. and Ruelle, D., Observables at infinity and states with short range correlations in statistical mechanics, Commun. Math. Phys. 13 (1969), 194-215.

[57] Lebowitz, J.L. and Penrose, O., Analytic and clustering properties of thermodynamic functions and distribution funcitnos for classical lattice and continuous systems, Commun. Math. Phys. 11 (1968), 99-124.

[58] Lieb, E.H., Exact solution of the problem of the entropy of two-dimensional ice, Phys. Rev. Lett. 18 (1967), 692-694.

[59] Liggett, T., Interacting Particle Systems, Springer-Verlag, New York 1985.

[60] Linnik, Yu.V., On the theory of nonhomogeneous Markov chains, Izvestiya AN SSSR, Seriya matematicheskaya 13:1 (1948), 65-94. [Russian]

[61] Malyshev, V.A. and Minlos, R.A., Gibbs Random Fields. The Method of Cluster Expansions, Kluwer, Dordrecht 1991.

[62] Malyshev, V.A. and Minlos, R.A., The Obituary. R.L. Dobrushin, Markov Processes and Related Fields 4 (1996), 1.

[63] Markov, A.A., A study of a general case of trials connected into a chain, Zapiski Akademii nauk po fiziko-matematicheskomu otdeleniya VIII seriya 25:3 (1910). [Russian] (Reprinted in: A.A. Markov, Izbrannye Trudy, Izdatel'stvo AN SSSR, Moskva (1951), 465-509).

[64] Minlos, R.A., The limiting Gibbs distribution, Functional Analysis and Its Appl. 1 (1967), 140-150.

[65] Minlos, R.A., The regularity of the limiting Gibbs distribution, Functional Analysis and Its Appl. 1 (1967), 206-217.

[66] Minlos, R.A., Lectures on statistical physics, Russian Mathematical Surveys - Uspekhi 23:1 (1968), 137-196.

[67] Minlos, R.A. and Sinai, Ya.G., Some new results on first order phase transitions in lattice gas models, Trans. of the Moscow Math. Society 17 (1967), 237-267.

[68] Minlos, R.A. and Sinai, Ya.G., The phase separation phenomenon at low temperature in some lattice models of a gas I, Math. of the USSR - Sbornik 73 (1967), 335-395.

[69] Minlos, R.A. and Sinai, Ya.G., The phase separation phenomenon at low temperature in some lattice models of a gas, Trans. of the Moscow Math Society 19 (1967), 121-196.

[70] Minlos, R., Shlosman, S. and Vvedenskaya, N., Memories of Roland Dobrushin, Notices of the AMS 43 (1996), 428-432.

[71] Morrey, C.B., On the derivation of the equation sof hydrodynamics from statistical mechanics, Comm. Pure Appl. Math. 8 (1955), 279-326.

[72] Mountford, T.S. and Prabhakar, B., On the weak convergence of departures from an infinite sequence of $\cdot / M / 1$ queues, Ann. Appl. Prob. 5 (1995), 121-127.

[73] Olla, S., Varadhan, S.R.S. and Yau, H.T., Hydrodynamical limit for a Hamiltonian system with weak noise, commun. Math. Phys. 155 (1993), 523-556.

[74] Onsager, L., Crystal statistics 1. A two-dimensional model with an order-disorder transition, Phys. Rev. 65 (1944), 117-149.

[75] Peierls, R., On the Ising model of ferromagnetism, Proc. of the Cambridge Phil. Soc. 32 (1932), 477-481. 
[76] Petrina, D.Ya. and Gerasimenko, V.I., Evolution of states of infinite systems in classical statistical mechanics, Soviet Scientific Reviews, Section C: Mathematical Physics Reviews 5 (1985), 1-52.

[77] Pirogov, S.A., Coexistence of phases for lattice models with several types of particles, Math. of the USSR - Izvestia 9 (1975), 1333-1357.

[78] Pirogov, S.A. and Sinai, Ya.G., Phase transitions of the first kind for small perturbations of the Ising model, Funct. Analysis and its Appl. 8 (1974), 21-25.

[79] Pirogov, S.A. and Sinai, Ya.G., Phase diagrams of classical lattice systems 1, Theoretical and Math. Physics 25 (1975), 1185-1192.

[80] Pirogov, S.A. and Sinai, Ya.G., Phase diagrams of classical lattice systems 2, Theoretical and Math. Physics 26 (1976), 39-49.

[81] Presutti, E., Pulvirenti, M., and Tirozzi, B., Time evolution of infinite classical systems with singular, long range, two body interactions, Commun. Math. Phys. 47 (1976), 85-91.

[82] Ruelle, D., Correlation functions of classical gases, Ann. Phys. 25 (1963), 109-120.

[83] Ruelle, D., Cluster property of the correlation functions of classical gases, Rev. Mod. Phys. 36 (1964), 580-584.

[84] Ruelle, D., States of classical statistical mechanics, J. Math. Phys. 8 (1967), 1657-1668.

[85] Ruelle, D., Statistical Mechanics, W.A. Benjamin, Reading, MA 1969.

[86] Ruelle, D., Superstable interactions in classical statistical mechanics, Commun. Math. Phys. 18 (1970), 127-159.

[87] Rybko, A.N. and Stolyar, A.L., On the ergodicity of random processes describing open service networks, Prob. of Inform. Trans. 28 (1992), 199-220.

[88] Sapogov, N.A., A limit theorem of Laplace-Lyapunov type for a singular Markov chain, Doklady AN SSSR 58:9 (1947), 29-38. [Russian]

[89] Shannon, C.E., A mathematical theory of communication, Bell System Tech. J., 27 (1948), 379-423, 623-656.

[90] Sherman, S., Markov random fields and Gibbs random fields, Israel J. Math. 14 (1973), $92-103$.

[91] Simon, B., The Statistical Mecahnics of Lattic Gases, I, Princeton University Press, Princeton 1993.

[92] Sinai, Ya.G., Ergodic properties of a gas of one-dimensional hard rods with an infinite number of degrees of freedom, Functional Analysis and its Appl. 6 (1972), 41-50.

[93] Sinai, Ya. G., Construction of dynamics in one-dimensional systems of statistical mechanics, Theoreticheskaya i Matematicheskaya Fizika 11 (1972), 248-258.

[94] Sinai, Ya.G., Theory of Phase Transitions: Rigorous Results, Pergamon Press, London 1982.

[95] Spitzer, F., Markov fields and Gibbs ensembles, Amer. Math. Monthly 78 (1971), 142-154.

[96] Spohn, H., Kinetic equations from Hamiltonian dynamics: Markovian limits, Rev. Mod. Phys. 52 (1980), 569-615.

[97] Spohn, H., Large Scale Dynamics of Interacting Particles, Springer-Verlag, New York 1991.

[98] Suhov, Yu., Professor Roland Dobrushin (the obituary), The Independent, November 29, 1995.

[99] Sullivan, W.G., Potentials for almost Markovian random fields, Commun. Math. Phys. 33 (1973), 61-74.

[100] Vvedenskaya, N.D. and Suhov, Y.M., The limiting departure flow in an infinite series of queues, Problems of Inform. Trans. 31 (1995), 100-111.

[101] Wulff, G., Zur Frage der Geschwindigkeit des Wachstums und der Auflösung der Kristallflachen, Z. Kryst. 34 (1901), 449-530. 


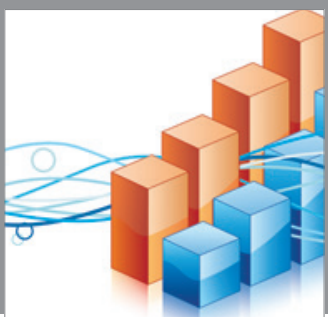

Advances in

Operations Research

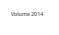

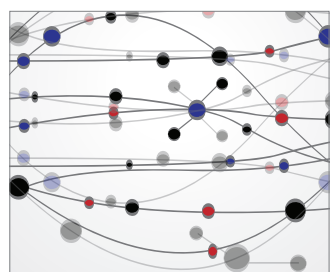

\section{The Scientific} World Journal
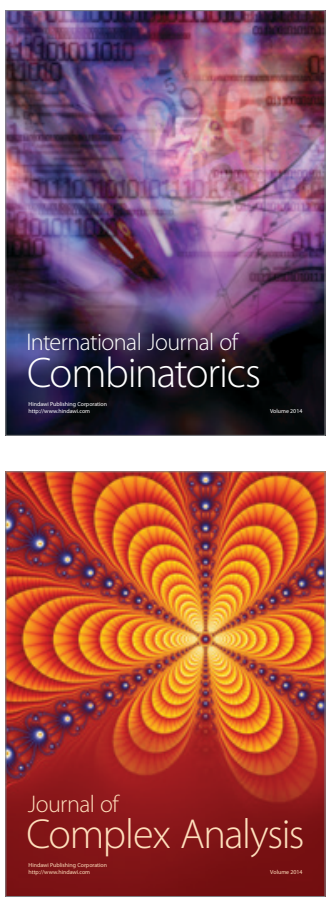

International Journal of

Mathematics and

Mathematical

Sciences
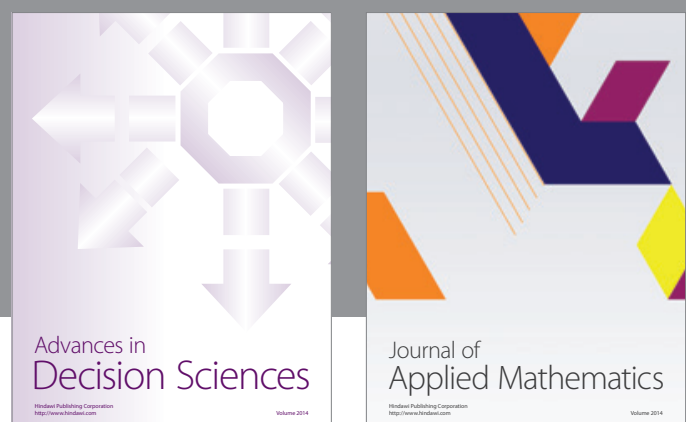

Journal of

Applied Mathematics
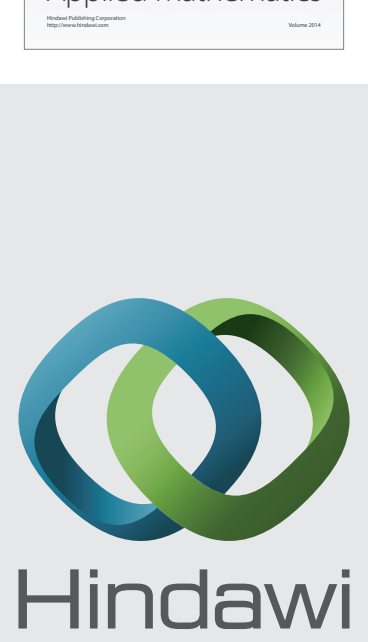

Submit your manuscripts at http://www.hindawi.com
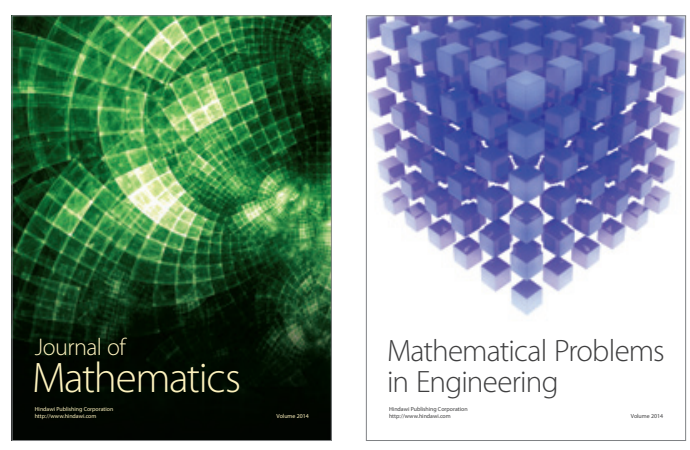

Mathematical Problems in Engineering
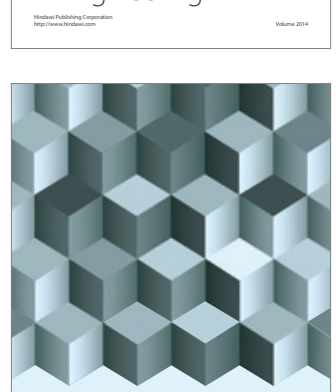

Journal of

Function Spaces
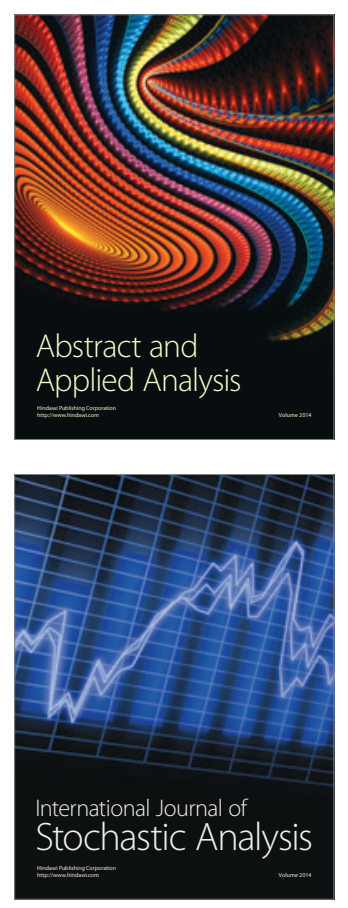

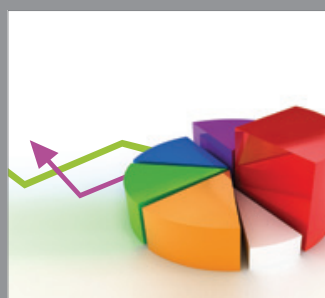

ournal of

Probability and Statistics

Promensencen
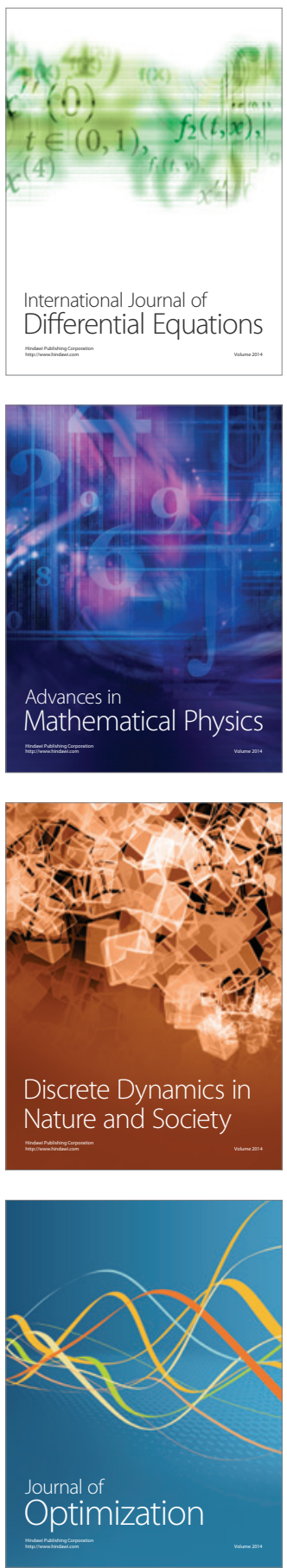